Ananth V. S.* and D. K. K. Vamsi

\title{
An Optimal Control Study with Quantity of Additional food as Control in Prey-Predator Systems involving Inhibitory Effect
}

https://doi.org/10.1515/cmb-2020-0121

Received March 21, 2021; accepted July 23, 2021

Abstract: Additional food provided prey-predator systems have become a significant and important area of study for both theoretical and experimental ecologists. This is mainly because provision of additional food to the predator in the prey-predator systems has proven to facilitate wildlife conservation as well as reduction of pesticides in agriculture. Further, the mathematical modeling and analysis of these systems provide the eco-manager with various strategies that can be implemented on field to achieve the desired objectives. The outcomes of many theoretical and mathematical studies of such additional food systems have shown that the quality and quantity of additional food play a crucial role in driving the system to the desired state. However, one of the limitations of these studies is that they are asymptotic in nature, where the desired state is reached eventually with time. To overcome these limitations, we present a time optimal control study for an additional food provided prey-predator system involving inhibitory effect with quantity of additional food as the control parameter with the objective of reaching the desired state in finite (minimum) time. The results show that the optimal solution is a bang-bang control with a possibility of multiple switches. Numerical examples illustrate the theoretical findings. These results can be applied to both biological conservation and pest eradication.

Keywords: Prey-predator systems; Inhibitory Effect; Additional food supplements; Optimal control problem; Bang-bang controls; Pontryagin's maximum principle

MSC: 49J30; 49K30; 92D25; 92D40; 92D45

\section{Introduction}

Prey-predator systems where predators are provided with additional food supplements are being extensively studied by biologists, ecologists (both theoretical and experimental ecologists) as well as mathematicians [16, 31, 33, 36, 46, 48]. This is mainly because the method of providing additional food not only effectively aids the conservation of threatened species [16, 30,31] and but also facilitates the control of harmful and invasive species $[23,47,48]$. Nonetheless, adequate care needs to be taken while providing additional food because lack of proper vigilance could lead to adverse consequences [30, 32]. These systems are generally modeled as differential equations and their dynamics are studied to analyse the behaviour of such systems. Some of the findings of mathematical studies and analysis of additional food provided prey-predator systems $[11,12,13,24,25,27,28,34,35,39,41]$ reveal that the additional food plays a crucial role in the dynamics of these systems, affecting the eventual state and stability of species. These findings are in line with the experimental observations when additional food is provided to the predators $[5,4,16,48]$

\footnotetext{
`Corresponding Author: Ananth V. S.: Department of Mathematics and Computer Science, Sri Sathya Sai Institute of Higher Learning, Prasanthi Nilayam, Puttaparthi, Anantapur District - 515134, Andhra Pradesh, India, E-mail: srini.ananth05@gmail.com

D. K. K. Vamsi: Department of Mathematics and Computer Science, Sri Sathya Sai Institute of Higher Learning, Prasanthi Nilayam, Puttaparthi, Anantapur District - 515134, Andhra Pradesh, India, E-mail: dkkvamsi@sssihl.edu.in
} 
In any mathematical model that describes a prey-predator interaction, one salient feature that characterizes the nature of this interaction is the Functional Response, which is defined as the rate at which prey is captured by the predator [17]. One of the many functional responses displayed by different predator species is the Type IV functional response where the predator's catchability reduces when prey density is high. This could be a consequence of prey interference or toxicity. In other words, the prey tend to exhibit group defense when in large densities as a survival mechanism $[7,10,49]$. The type IV functional response is also called the functional response involving inhibitory effect. For example, caterpillar species Malacosoma disstria display group defense against their natural enemy parasitoids to reduce risk of predation [22]. As a result of forming bigger groups, the parasitoid does not succeed in catching its prey in spite of multiple attacks.

Recently, in [40] and [45], the authors have modeled and studied the following additional food system involving type IV functional response

$$
\begin{aligned}
& \dot{N}=r N\left(1-\frac{N}{K}\right)-\left(\frac{c N}{(A \eta \alpha+a)\left[b N^{2}+1\right]+N}\right) P, \\
& \dot{P}=e\left(\frac{N+\eta A\left(b N^{2}+1\right)}{(A \eta \alpha+a)\left[b N^{2}+1\right]+N}\right) P-m P,
\end{aligned}
$$

where $\mathrm{N}$ denotes the density of the prey species, $\mathrm{P}$, the density of predator species and $\mathrm{A}$, the biomass of additional food provided to the predators. The biological meaning of the parameters of the system (1.1) - (1.2) are given in the following table:

\begin{tabular}{l|l}
\hline Parameters & Biological Meaning \\
\hline$r$ & Intrinsic Growth rate of the prey \\
\hline$K$ & Prey carrying Capacity \\
\hline$m$ & Mortality rate of predators in the absence of prey \\
\hline$e_{1}$ & Search time of the predator per unit prey availability \\
\hline$e_{2}$ & Search time of the predator per unit additional food \\
\hline$h_{1}$ & Handling time of predator per prey item \\
\hline$h_{2}$ & Handling time of predator per unit additional food \\
\hline$b$ & Inhibitory effect of the prey \\
\hline$c=\frac{1}{h_{1}}$ & Maximum rate of predation in the absence of inhibitory effect \\
\hline$a=\frac{1}{\sqrt{e_{1} h_{1}}}$ & Half-Saturation rate of Predators in the absence of inhibitory effect \\
\hline$\epsilon_{1}, 0<\epsilon_{1}<1$ & Nutritional Value of the prey \\
\hline$\epsilon_{2}, 0<\epsilon_{2}<1$ & Nutritional Value of the additional food \\
\hline$e=\epsilon_{1} c=\frac{\epsilon_{1}}{h_{1}}$ & Maximum growth rate of predators due to consumption of prey \\
\hline$\alpha=\frac{\epsilon_{1} / h_{1}}{\varepsilon_{2} / h_{2}}$ & Ratio of Maximum growth rate of predators due to prey with additional food \\
\hline$\eta=\frac{e_{2} \epsilon_{2}}{e_{1} \epsilon_{1}}$ & $\begin{array}{l}\text { Ratio of search time of predator per unit food item of additional food and prey } \\
\text { relative to nutritional values of additional food and prey }\end{array}$ \\
\hline
\end{tabular}

The parameter $\alpha$ denotes the efficiency of predator to convert the additional food available into predator biomass relative to conversion of prey. $\alpha$ is inversely proportional to the nutritional value of the additional food and directly proportional to the handling time of the additional food. In this study, we use the parameter $\alpha$ to represent the Quality of additional food provided to the predators. Additional food is termed high quality if $\alpha<\frac{\beta}{\delta}$ and low quality otherwise. The parameter $\eta=\frac{e_{2}}{e_{1}} \frac{\epsilon_{2}}{\epsilon_{1}}$ denotes the ratio of search time of predator per unit food item of additional food and prey relative to nutritional values of additional food and prey. The term $\eta A=\frac{e_{2}}{e_{1}}(A) / \frac{\epsilon_{1}}{\epsilon_{2}}$ represents the quantity of additional food discernible to the predator with respect to the prey relative to the nutritional value of prey to the additional food. 
Let $\mathrm{N}=\mathrm{ax} ; \mathrm{t}=\mathrm{rT} ; \mathrm{P}=\mathrm{y} \frac{r a}{c}$; which gives $d N=a d x ; \frac{d t}{r}=d T ; d P=\frac{r a}{c} d y$. Now, using the following transformations $\gamma=\frac{K}{a}, \quad \omega=b a^{2}, \quad \beta=\frac{e}{r}, \quad \delta=\frac{m}{r}$, and taking $\xi=\eta \frac{A}{a}$, we get the corresponding non-dimensionalized system as follows:

$$
\begin{aligned}
& \frac{d x}{d t}=x\left(1-\frac{x}{\gamma}\right)-\left[\frac{x y}{(1+\alpha \xi)\left(\omega x^{2}+1\right)+x}\right] \\
& \frac{d y}{d t}=\beta\left[\frac{x+\xi\left(\omega x^{2}+1\right)}{(1+\alpha \xi)\left(\omega x^{2}+1\right)+x}\right] y-\delta y
\end{aligned}
$$

In the above non-dimensionalised system, $\gamma$ and $\omega$ are parameters that represent the carrying capacity and inhibitory effect respectively. In this study, we consider the parameter $\xi$ to denote the Quantity of additional food provided to the predators. Let $f(x)=\frac{x}{(1+\alpha \xi)\left(\omega x^{2}+1\right)+x}$ and $g(x)=\left(1-\frac{x}{\gamma}\right)\left((1+\alpha \xi)\left(\omega x^{2}+1\right)+x\right)$. Then, the system (1.3) - (1.4) can be modified as follows:

$$
\begin{aligned}
& \frac{d x}{d t}=(g(x)-y) f(x) \\
& \frac{d y}{d t}=\left(\beta f(x)\left[1+\frac{\xi}{x}\left(\omega x^{2}+1\right)\right]-\delta\right) y
\end{aligned}
$$

The outcomes of the works [40] and [45] concede the importance of quality and quantity of additional food that is provided to the predator reinforcing the inferences from the studies $[18,19,30]$ and $[50]$. However, in [40] the desired states are shown to be reached eventually with time as asymptotes. This makes the outcomes not that practically helpful. To overcome this limitation, we propose to study the system (1.3) (1.4) further in the direction of achieving controllability in minimum (finite) time. Also, we see from various ecological studies that the quantity of additional food plays a significant role in the dynamics of the system $[4,44,47,48,50]$. Since the nutritional value and the conversion factor are always fixed for a given food item, the quantity of consumption thus plays a major role in determining the effect of providing the additional food. Also, it is practically easier to vary the quantity of food supplements provided than varying the quality. Therefore, the study of controllability using the quantity of additional food becomes relevant.

Motivated by the above discussion, in this article, we study the role of quantity of additional food $\xi$ in influencing the global dynamics of the additional food provided system (1.3) - (1.4) in achieving the desired state in minimum (finite) time. To that end, we first determine the possible admissible states that could be reached by varying the quantity in the range $\left[\xi_{\min }, \xi_{\max }\right]$. We then formulate and study a Time optimal control problem using quantity of additional food as the control parameter. We use Filippov's Existence Theorem to prove the existence of optimal solution and Pontryagin's Maximum Principle to obtain the characteristics of the optimal solutions. Throughout the study, we keep the quality of additional food $\alpha$ fixed. The outcomes of this work can benefit eco-managers in providing additional food to species depending on the objective being biological conservation or pest management. Optimal control studies for additional food provided type II functional response have been done in the works [37, 38]. Since type IV response is a more general form of type II response, it becomes significant to perform control studies on systems involving type IV response. The controllability studies with respect to quality of additional food keeping the quantity fixed has its own relevance and is under progress [1].

The section-wise division of this article is as follows: In the next section, we discuss the biological relevance of having quantity as the control parameter. In section 3, we study the role of quantity of additional food and its influence on the dynamics of the type IV additional food provided system and determine the possible admissible equilibria. In section 4, we formulate and study the time optimal control problem with quantity of additional food as the control parameter following which in section 5 , we discuss optimal solution strategies and applications to pest management. Then, in section 6, we illustrate the theoretical results using numerical examples followed by a section on the role of inhibitory effect. Finally, we present the discussion and conclusions in section 8 . 


\section{Biological Relevance of Quantity as a Control Parameter}

The findings of several ecological and entomological studies supported by experimental observations show that the quantity of additional food provided to the predator species impact their survival, oviposition rate, longevity, fecundity as well as predation rate $[2,4,14,29,44,47,48,50,51]$. This is more so applicable in the context of bio-control or pest management where natural enemies of these invasive species or pests are mass reared for attacking their target prey as a part of habit management [20]. Plutella xylostella (diamondback moth) is a herbivore whose parasitoid is Diadegma semiclausum. The parasitoid D. semiclausum was tested with nine sugars and honey dew sources to provide carbohydrate rich food in its adult stages [50]. The gustatory response of $D$. semiclausum was found to be high when their concentration was $1 \mathrm{M}$ and the response started to drop with decreasing concentration.

Macrolophus pygmaeus is an omnivorous mirid predator which is one of the widely used natural enemies in augmentative biological control against pests like whiteflies, arthropod pests, tomato pests etc., in Europe [29]. Ephestia kuehniella eggs are a factitious food which are used as an alternate source to rear these species. The results from the experimental studies [47] reveal that as the number of $E$. kuehniella eggs provided per three days increase from 10 to 40, the M. pygmaeus species reduced their development time significantly, females had better oocyte counts and nymphs increased their survival. However, it cannot be concluded that high quantity of additional food supplements is always beneficial. For instance, Nesidiocoris tenuis, which is a zoophytophagus natural enemy of tomato pest Tuta absoluta, is provided with sugars of concentration $0.5 \mathrm{M}$ and $1 \mathrm{M}$, it is found that the immature furvival of $N$ tenuis was lower when provided with $1 \mathrm{M}$ sucrose than with $0.5 \mathrm{M}$ sucrose [43].

Thus, the influence of quantity of additional food supplements on predator species (and hence on the ecosystem) depends on the quality of additional food [48]. Since the quality of additional food defined above not just implies the calorific value of the item but the handling time and the relative efficiency, the predators response to a specific item also plays a major role in determining the quality of additional food which can be treated constant for a specific item. Thus, using quantity of additional food to study the controllability of the system very pertinent to understand the dynamics of the system and also to drive the system to a certain desired state in minimum time.

\section{Role of Quantity in the Global Dynamics of the Additional food system}

In this section, we will outline the global dynamics of the additional food system (1.3) - (1.4) and the role of quantity of additional food in influencing the eventual state and stability of the system. The type IV additional food provided system (1.3) - (1.4) admits four equilibrium points: the trivial equilibrium $E_{0}^{\star}=$ $(0,0)$, the axial equilibrium point $E_{1}^{\star}=(\gamma, 0)$, and two interior equilibria $E_{2}^{\star}=\left(x_{1}^{\star}(\alpha, \xi), y_{1}^{\star}(\alpha, \xi)\right)$ and $E_{3}^{\star}=$ $\left(x_{2}^{\star}(\alpha, \xi), y_{2}^{\star}(\alpha, \xi)\right)$ given by

$$
\begin{aligned}
& E_{2}^{\star}=\left(x_{1}^{\star}, y_{1}^{\star}\right)=\left(\frac{(\beta-\delta)-\sqrt{(\beta-\delta)^{2}-4 \omega[\delta(1+\alpha \xi)-\beta \xi]^{2}}}{2 \omega[\delta(1+\alpha \xi)-\beta \xi]}, g\left(x_{1}^{\star}\right)\right), \\
& E_{3}^{\star}=\left(x_{2}^{\star}, y_{2}^{\star}\right)=\left(\frac{(\beta-\delta)+\sqrt{(\beta-\delta)^{2}-4 \omega[\delta(1+\alpha \xi)-\beta \xi]^{2}}}{2 \omega[\delta(1+\alpha \xi)-\beta \xi]}, g\left(x_{2}^{\star}\right)\right) .
\end{aligned}
$$


Table 1: Summary of dynamics under Condition I for the additional food provided system (1.3) - (1.4)

\begin{tabular}{l|l|l|l|l|l|l}
\hline \multicolumn{3}{c|}{ Figure and Region } & \multicolumn{4}{c}{ Nature of the equilibria } \\
\hline \hline Fig. 2 & Fig. 3 & Fig. 4 & $E_{0}^{\star}$ & $E_{1}^{\star}$ & $E_{2}^{\star}$ & $E_{3}^{\star}$ \\
\hline A1 & B1 & C1 & Saddle & Stable & - & - \\
\hline- & B2 & C2 & Saddle & Stable & Stable & Saddle \\
\hline A2 & B3 & - & Saddle & Stable & $\begin{array}{l}\text { Unstable } \\
\text { GAS Limit Cycle } \\
\text { Homoclinic Orbit }\end{array}$ & Saddle \\
\hline A3 & B5 & - & Saddle & Saddle & $\begin{array}{l}\text { Unstable } \\
\text { GAS Limit Cycle }\end{array}$ & - \\
\hline A4 & B6 & C3 & Saddle & Saddle & Stable & - \\
\hline A5 & B7 & C4 & Unstable & Saddle & - & - \\
\hline A6 & B8 & C5 & Saddle & Stable & - & - \\
\hline
\end{tabular}

The interior equilibria exist only when the term under the square root $(\beta-\delta)^{2}-4 \omega[\delta(1+\alpha \xi)-\beta \xi]^{2}>0$ and $x_{1}^{\star}<\gamma$ and $x_{2}^{\star}<\gamma$. The analysis carried out in the works [40] and [45] show that the dynamics of the system (1.3) - (1.4) depend on the dynamics of the initial system (without additional food) and nature of its isoclines. Also, for maintaining brevity, we too shall confine this work to Condition I of the initial system as considered in [40], where interior equilibrium is not admitted by the initial system. In other words, we consider the following condition holding true:

$$
\omega-\frac{(\beta-\delta)^{2}}{4 \delta^{2}}>0
$$

Under this condition, we get three different cases for the additional food system. The figure 1 depicts the different types of the prey isocline that occur under the condition - I.
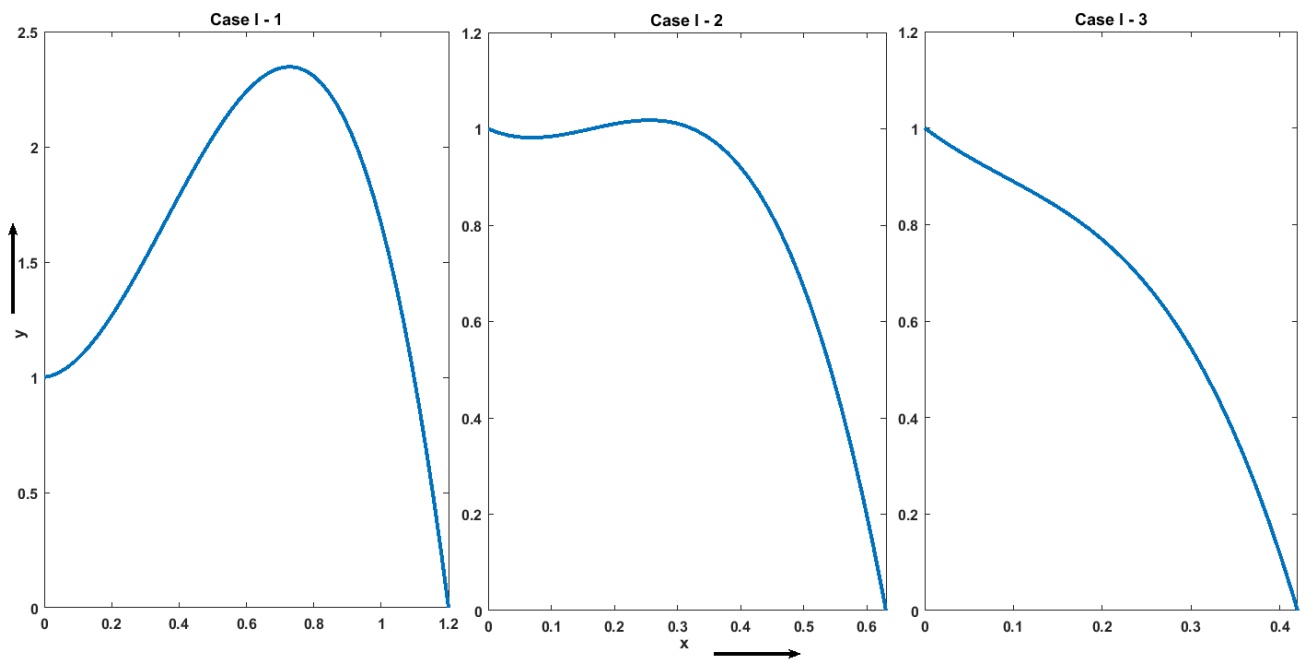

Figure 1: This figure shows the nature of prey isocline curve of the initial system under the three sub-cases of condition - I. These are plotted for the values of parameters $\beta=2.2$ and $\delta=0.4$ 


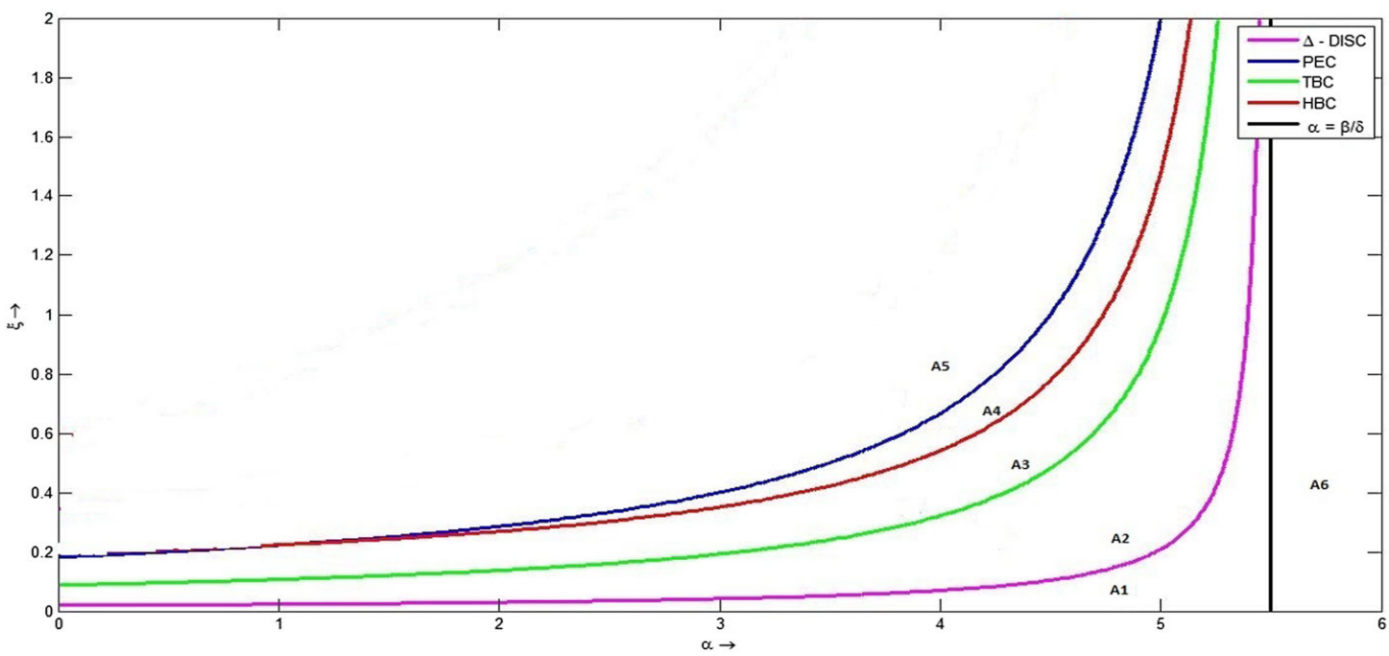

Figure 2: This figure shows the $\alpha \xi$ - space being divided by the Discriminant curve (DISC), Prey Elimination Curve (PEC) and the two bifurcation curves: the Hopf bifurcation curve (HBC), the transcritical bifurcation curve (TBC) and the curve $\alpha=\frac{\beta}{\delta}$. The parameter values used are $\beta=2.2, \delta=0.4, \gamma=1.2$, and $\omega=8$, satisfying the condition I-1

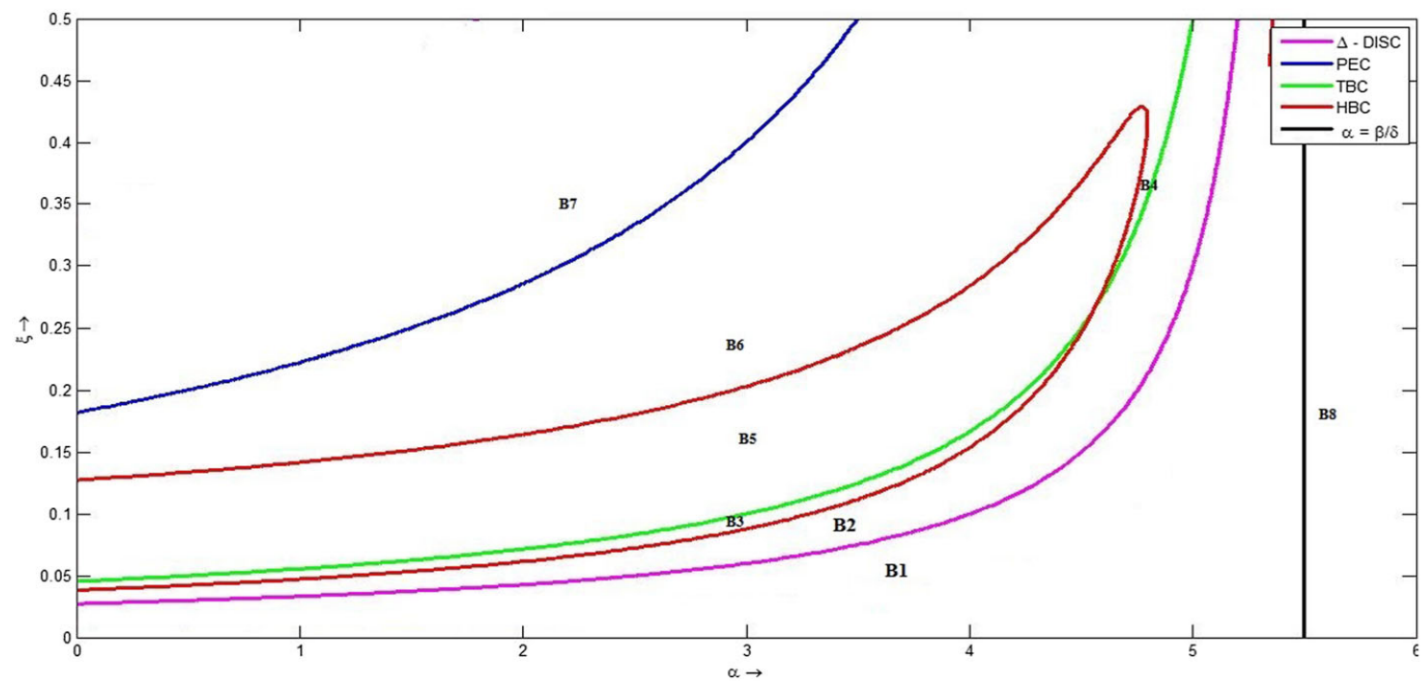

Figure 3: This figure shows the $\alpha \xi$ - space being divided by the Discriminant curve (DISC), Prey Elimination Curve (PEC) and the two bifurcation curves: the Hopf bifurcation curve (HBC), the transcritical bifurcation curve (TBC) and the curve $\alpha=\frac{\beta}{\delta}$. The parameter values used are $\beta=2.2, \delta=0.4, \gamma=0.63$, and $\omega=7$, satisfying the condition I-2

The phase space analysis of the additional food system reveals that along with the nature of the prey isocline curve (as shown in figure 1), the eventual state of the system and stability of the equilibria also depends on the values assumed by the two parameters $\alpha$ and $\xi$ with respect to the three bifurcation curves and the discriminant curve given below:

The Prey Elimination Curve (PEC)

$$
\beta \xi-\delta(1+\alpha \xi)=0
$$

the Transcritical Bifurcation Curve (TBC)

$$
\beta\left(\gamma+\xi\left(\omega \gamma^{2}+1\right)\right)-\delta\left((1+\alpha \xi)\left(\omega \gamma^{2}+1\right)+\gamma\right)=0
$$




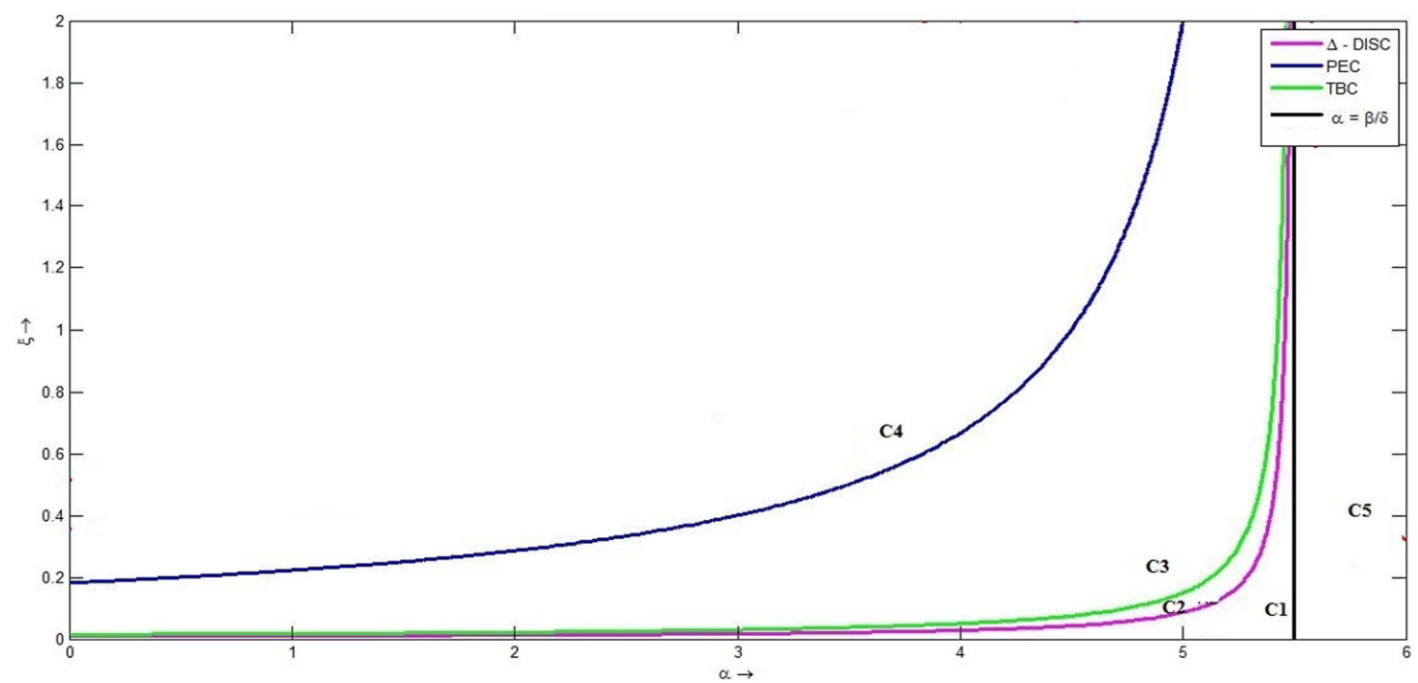

Figure 4: This figure shows the $\alpha \xi$ - space being divided by the Discriminant curve (DISC), Prey Elimination Curve (PEC) and the two bifurcation curves: the Hopf bifurcation curve (HBC), the transcritical bifurcation curve (TBC) and the curve $\alpha=\frac{\beta}{\delta}$. The parameter values used are $\beta=2.2, \delta=0.4, \gamma=0.42$, and $\omega=6.4$, satisfying the condition I-3

the Hopf Bifurcation Curve (HBC)

$$
\begin{aligned}
& 3 \omega(1+\alpha \xi)\left[\frac{(\beta-\delta)-\sqrt{(\beta-\delta)^{2}-4 \omega[\delta(1+\alpha \xi)-\beta \xi]}}{2 \omega[\delta(1+\alpha \xi)-\beta \xi]}\right]^{2} \\
& +(2-2 \omega \gamma(1+\alpha \xi))\left[\frac{(\beta-\delta)-\sqrt{(\beta-\delta)^{2}-4 \omega[\delta(1+\alpha \xi)-\beta \xi]}}{2 \omega[\delta(1+\alpha \xi)-\beta \xi]}\right] \\
& +(1+\alpha \xi)-\gamma=0
\end{aligned}
$$

and the Discriminant Curve (DISC)

$$
(\beta-\delta)^{2}-4 \omega(-\beta \omega+\delta(1+\alpha \xi))^{2}=0
$$

The table 1 summarizes the global dynamics of the additional food system (1.3) - (1.4). The figures (2), (3), and (4) (which are called bifurcation diagrams) depict how the four curves along with the curve $\alpha=\frac{\beta}{\delta}$ divide the $(\alpha, \xi)$ - space for each of the sub-conditions I-1, I-2 and I-3 respectively. Since the optimal control studies are based on the quantity of additional food as the control parameter, we wish to perceive the global dynamics from the point of view of quantity of additional food. The Table - 2 shows the global dynamics of the additional food system (1.3) - (1.4) using quantity of additional food as focus.

In the previous sections, we saw that the quality of additional food can be classified into high quality and low quality depending on the position of the parameter $\alpha$ with respect to $\frac{\beta}{\delta}$. From the discussion above, we see that the additional food system does not admit any interior equilibria when the additional food is provided of low quality under the Condition - I. This is due to the dynamics established in [40] which states that: If the type IV system does not admit interior equilibrium in the absence of additional food, then the additional food system shall never admit interior equilibria by providing low quality additional food $\alpha>\frac{\beta}{\delta}$. Thus, in this work, we will discuss only the provision of high quality additional food when the system has no interior equilibrium in the absence of additional food. 


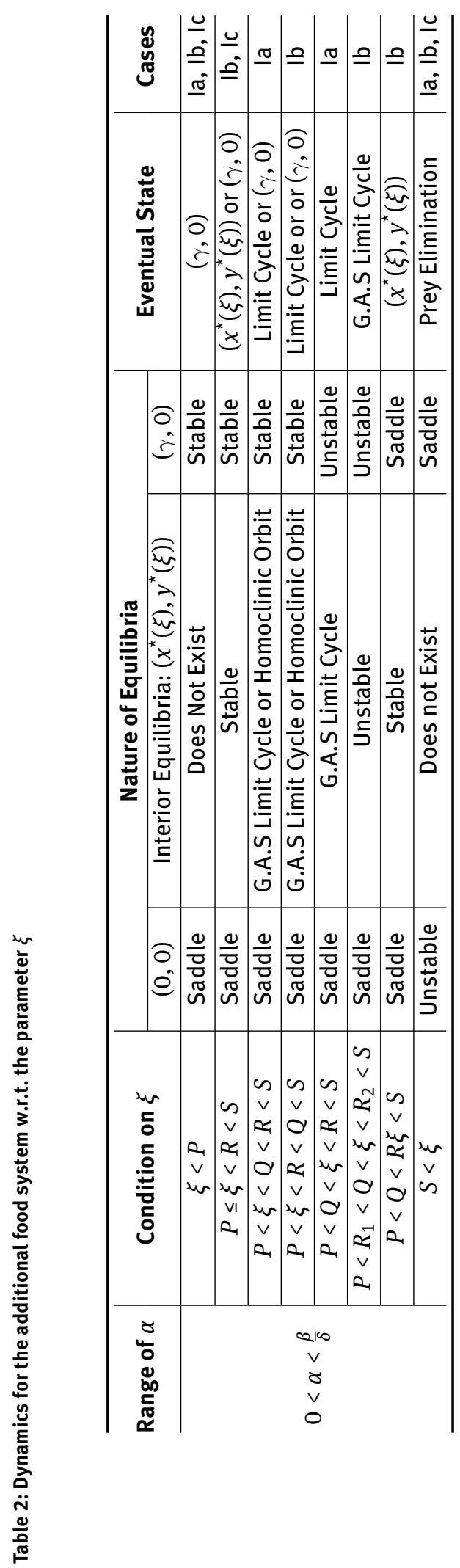

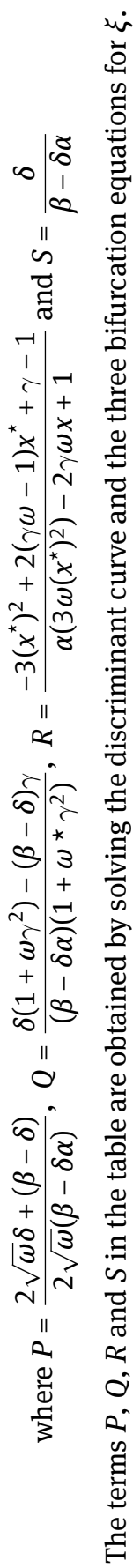


From the table - 2, we see that by providing appropriate quantity of high quality additional food, the system can be driven to any desired state. We will now determine the admissible states that can be reached in finite time when additional food of high quality is provided and by varying the quantity in the range $\left[\xi_{\min }, \xi_{\max }\right]$, where $\xi_{\min }\left(\xi_{\max }\right)$ represents the lowest (highest) quantity of additional food provided. We observe from the table - 2 that in the context of pest eradication or pest management, where the terminal state eliminates prey (or maintains prey density with least harm to the system), we need to have $\xi>\frac{\beta}{\beta-\delta \alpha}$, so that the trajectories which emerge from below the prey isocline curve touch the y-axis in finite time [37, 40] (or reach the desired state sufficiently close to $x=0$ ). Otherwise, there is no possibility of any trajectory that moves towards the predator axis (y-axis). In some cases of bio-control it is also preferred that the prey (pest) are not completely eliminated from the system because predator tends to damage the crops in the ecosystem after the target prey gets eliminated [6, 43]. In such cases, prey are maintained at low densities such that they do not damage crops. Based on the discussion above, we will now state a result in the context of pest management:

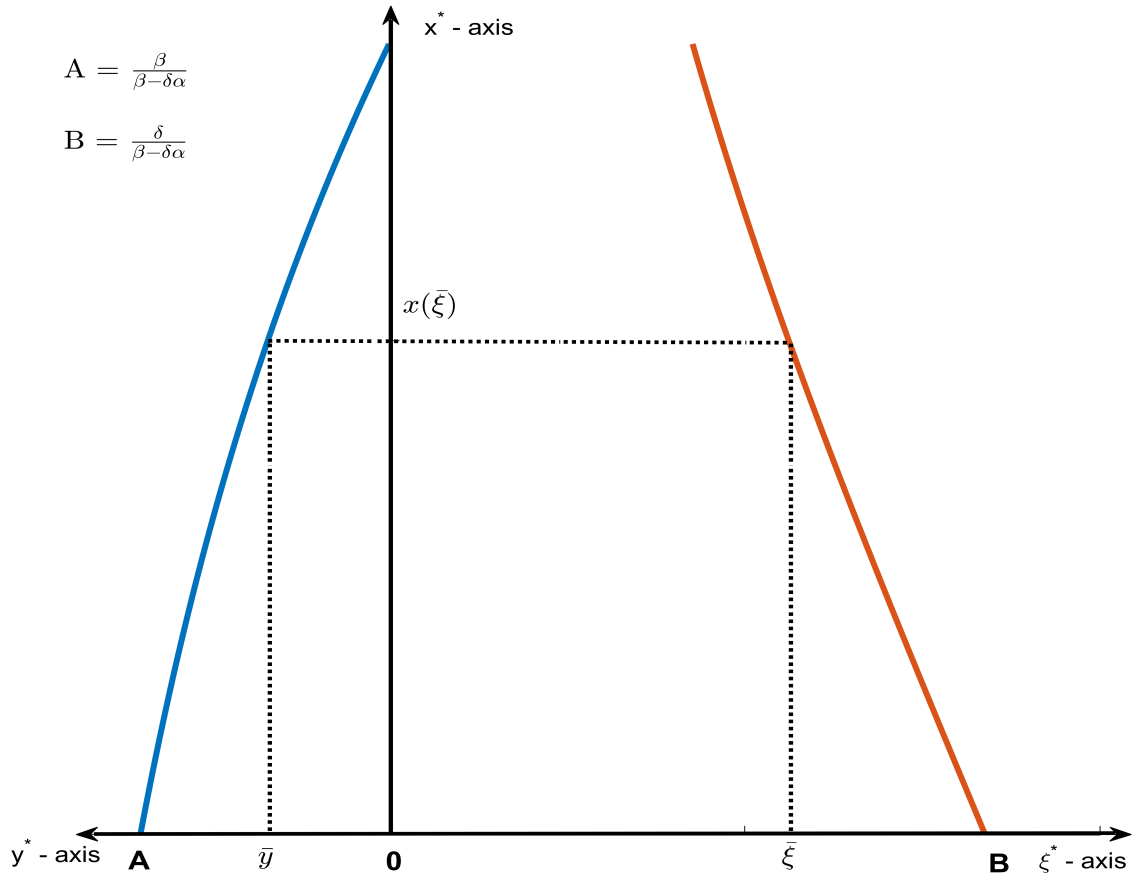

Figure 5: Admissible Equilibria and Control - Case I

Proposition 1. If additional food provided to the predators is of high quality satisfying $\beta-\delta \alpha>0$, then the pest can be eliminated, provided the quantity of additional food supply satisfies $\xi>\frac{\delta}{\beta-\delta \alpha}$.

Proof. From the dynamics presented in the table - 2, we observe that when $\beta-\delta \alpha>0$ and $\xi>\frac{\delta}{\beta-\delta \alpha}$, the trivial equilibrium is unstable and the axial equilibrium $(\gamma, 0)$ is a saddle whose unstable manifold moves towards origin on the prey axis.

Thus, for any solution initiated under the prey isocline curve, the saddle of axial equilibrium pushes the trajectory towards origin whose unstable nature in turn drives the system asymptotically towards the predator axis eventually eliminating the prey. On the other hand, for the solutions generating above the prey isocline curve, the prey isocline curve itself acts as an unstable manifold of the axial equilibrium thereby driving the state towards prey elimination. 


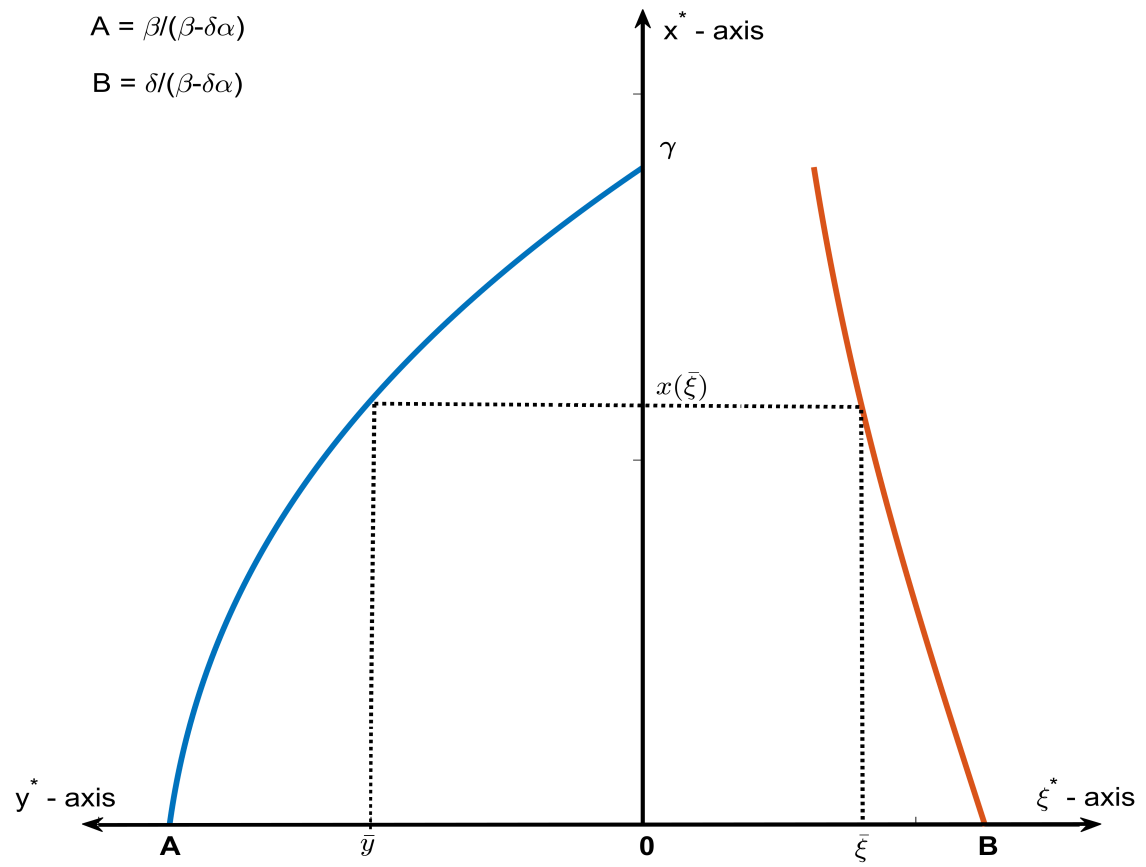

Figure 6: Admissible Equilibria and Control - Case II

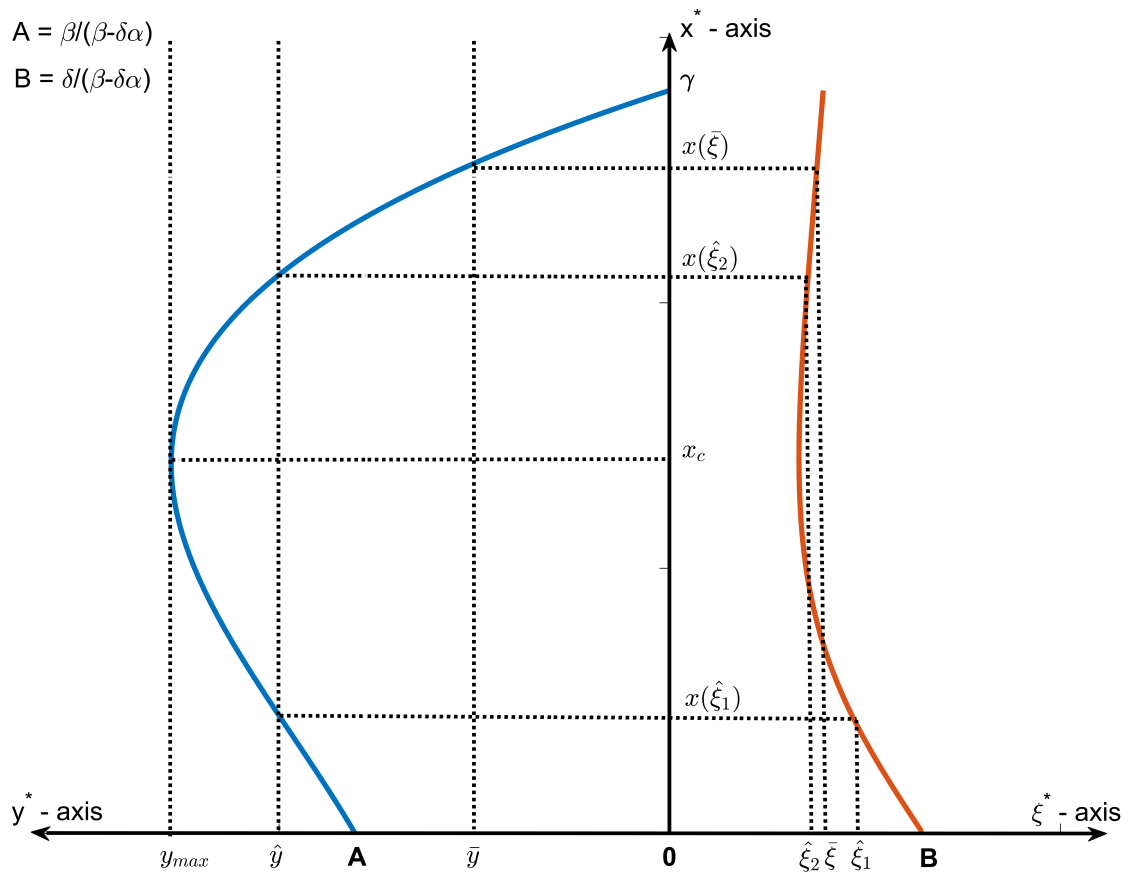

Figure 7: Admissible Equilibria and Control - Case III

Let us now consider the case of co-existence of species in the additional food system. With that goal, we must drive the additional food system towards interior equilibrium point which would be admissible. Since we see from the dynamics of the system (1.3) - (1.4) given in table - 1 that second interior equilibrium is a saddle throughout its existence, we do not wish to drive the system to that equilibrium point. However, from the table 2, we see that under certain conditions, there is a possibility of occurence of a homoclinic orbit 


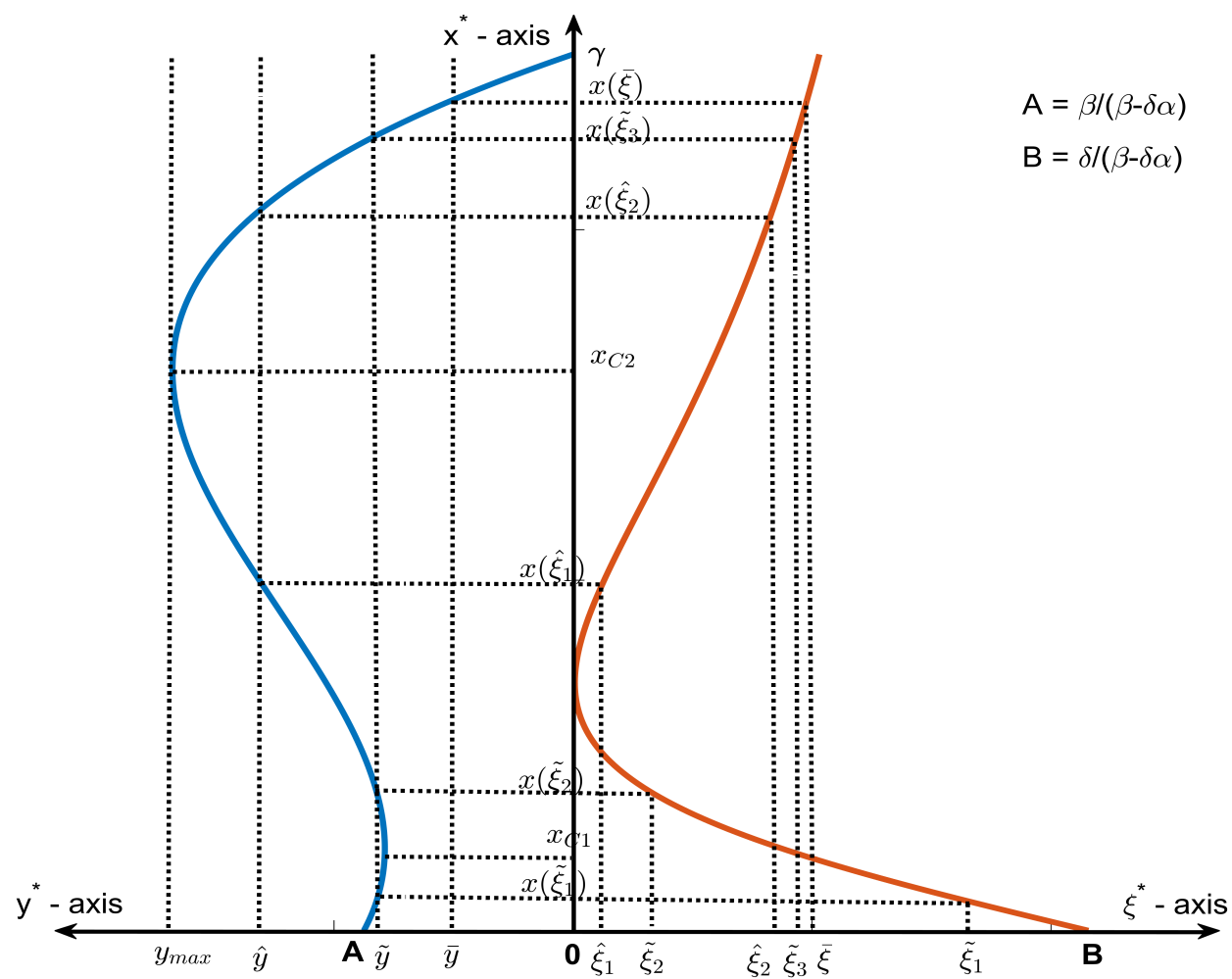

Figure 8: Admissible Equilibria and Control - Case IV

especially when the interior equilibrium $E_{2}^{*}$ is unstable. Under those conditions, it may not be possible to reach $E_{2}^{\star}$ from any initial point. Thus, we will consider $E_{2}^{\star}$ to be admissible only when it is unique, i.e., when the prey component of $E_{3}^{\star}=\left(x_{3}, y_{3}\right)$ is such that $x_{3}>\gamma$. Henceforth, we will refer to $E_{2}^{\star}=\left(x^{\star}(\xi), y^{\star}(\xi)\right)$ as the interior equilibrium. Let us consider the interior equilibrium $E_{2}^{\star}=\left(x^{\star}(\xi), y^{\star}(\xi)\right)$ for a fixed $\xi>0$. The prey and predator component are given by

$$
\begin{aligned}
& x^{\star}(\xi)=\frac{(\beta-\delta)-\sqrt{(\beta-\delta)^{2}-4 \omega[\delta(1+\alpha \xi)-\beta \xi]^{2}}}{2 \omega[\delta(1+\alpha \xi)-\beta \xi]} \\
& y^{\star}=\left(1-\frac{x^{\star}(\xi)}{\gamma}\right)\left(x^{\star}(\xi)+(1+\alpha \xi)\left(1+\omega\left(x^{\star}(\xi)\right)^{2}\right)\right)
\end{aligned}
$$

Now, solving (3.1) for $\xi$, we get

$$
\xi=\frac{\delta\left(1+\omega\left(\chi^{\star}(\xi)\right)^{2}\right)-\chi^{\star}(\xi)(\beta-\delta)}{(\beta-\delta \alpha)\left(1+\omega\left(x^{\star}(\xi)\right)^{2}\right)}
$$

Substituting for $\xi$ in (3.2) using (3.3), we get

$$
y^{\star}(\xi)=\left(\frac{\beta}{\beta-\delta \alpha}\right)\left(1-\frac{x^{\star}(\xi)}{\gamma}\right)\left[1+\omega\left(x^{*}(\xi)\right)^{2}+x^{\star}(\xi)(1-\alpha)\right]
$$

The above equation (3.4) gives us the set of all admissible equilibrium points for the additional food system (1.3) - (1.4). This curve (3.4) intersects the $y^{\star}$ - axis at $y^{\star}=\frac{\beta}{\beta-\delta \alpha}$. In order to understand the nature of the curve in (3.4), we consider the first derivative of $y^{\star}(\xi)$ with respect to $x^{\star}(\xi)$ :

$$
\frac{d y^{\star}(\xi)}{d x^{\star}(\xi)}=\frac{\beta}{\gamma(\beta-\delta \alpha)}\left[-3 \omega\left(x^{\star}(\xi)\right)^{2}+2(\omega+\alpha-1) x^{\star}(\xi)+\gamma(1-\alpha)-1\right]
$$


By obtaining the roots of the quadratic equation $-3 \omega\left(x^{*}(\xi)\right)^{2}+(2 \omega+\alpha-1) x^{*}(\xi)+\gamma(1-\alpha)-1=0$, we get the critical points of the admissible curve of all equilibria and based on that we can determine the nature of admissible points. The roots of the above quadratic equation are given by

$$
\begin{aligned}
& x_{C 1}=\frac{2(\omega \gamma-\alpha-1)-\sqrt{12(\gamma(1-\alpha)-1)+(2 \omega \gamma+2 \alpha-2)^{2}}}{6 \omega} \\
& x_{C 2}=\frac{2(\omega \gamma-\alpha-1)+\sqrt{12(\gamma(1-\alpha)-1)+(2 \omega \gamma+2 \alpha-2)^{2}}}{6 \omega}
\end{aligned}
$$

Depending on the existence of the above roots we get the following four cases of the nature of the admissible curve (3.4):

(i) Case I: The roots of the equation are non-real and thus the curve (3.4) is monotonically decreasing (refer to figure - 5).

(ii) Case II: The roots of the equation are real and exist but both are negative. Thus the curve (3.4) is monotonically decreasing (refer to figure - 6).

(iii) Case III: The roots of the equation are real and one root is positive while the other is negative. Thus, the curve (3.4) has a crest which is a point of maxima (refer to figure - 7).

(iv) Case IV: The roots of the equation are real and both roots are positive. Thus, the curve (3.4) has both a crest and a trough in the positive quadrant (refer to figure - 8).

Depending on the nature of the admissible curve (3.4), for a chosen predator density, we may have one or more prey density options to drive the system to, depending on the quantity of additional food provided. We summarize them in the following result:

Proposition 2. For Prey Species Conservation:

Let the equilibrium $E_{2}^{\star}$ be the unique interior equilibrium for the system (1.3) - (1.4). Then, for $0<x<\gamma$, there exists a unique $\xi$ such that $(x, y(\xi))$ is an admissible interior equilibrium for the system (1.3) - (1.4).

For Predator Species Conservation:

Let the equilibrium $E_{2}^{\star}$ be the unique interior equilibrium for the system (1.3) - (1.4). Let $A=\frac{\beta}{\beta-\delta \alpha}$ and let $x_{C}$ denote the critical point in Case III with the corresponding predator component $y_{\max }$. Let $x_{C 1}$ and $x_{C 2}$ be the two positive critical points in Case IV whose predator components are denoted by $y_{\min }$ and $y_{\max }$ respectively.

1. For every $0<\bar{y}<\min \left\{A, y^{\star}\left(x_{C 1}\right)\right\}$, there exists a unique value of quantity, $\bar{\xi}$ such that $(x(\bar{\xi}), \bar{y})$ is an admissible equilibria for the system (1.3) - (1.4) (refer to figures 5 - 8).

2. For $\hat{y}$ such that $A<\hat{y}<y_{\max }$, there exist two choices of quantity $\hat{\xi}_{1}$ and $\hat{\xi}_{2}$ such that $\left(x\left(\hat{\xi}_{i}\right), \hat{y}\right)$ for $i=1,2$ are admissible equilibria for the system (1.3) - (1.4) (refer to figures 7 and 8).

3. For $\tilde{y}$ such that $y_{\min }<\tilde{y}<A$, there exist three choices of quantity $\tilde{\xi}_{1}, \tilde{\xi}_{2}$ and $\tilde{\xi}_{3}$ such that $\left(x\left(\tilde{\xi}_{i}\right), \tilde{y}\right)$ for $i=1,2,3$ are admissible equilibria for the system (1.3) - (1.4) (refer to figure 8).

\section{Time Optimal Control Studies}

We will now formulate and study a time optimal control problem for the additional food provided system (1.3) - (1.4) that would drive the system from a given initial state $\left(x_{0}, y_{0}\right)$ to the desired terminal state $(\bar{x}, \bar{y})$ in minimum time using optimal quantity of additional food provided to the predator species, with for a fixed quality of additional food. Let $\alpha>0$ be fixed, and let the parameter representing the quantity of 
additional food be the control function $\xi(t)$ whose range is in the interval $\left[\xi_{\min }, \xi_{\max }\right]$. Then the time optimal control problem (a Mayer Problem of Optimal Control [8]) can be defined for the system (1.3) - (1.4) as follows:

$$
\begin{cases}\min _{\xi_{\min } \leq \xi(\mathrm{t}) \leq \xi_{\max }} \mathbf{T} & \\ \text { subject to: } & \text { Optimal Control Problem } \\ \dot{x}=f_{1}(x, y, \xi) & \\ \dot{y}=f_{2}(x, y, \xi) & \\ (x(0), y(0))=\left(x_{0}, y_{0}\right) \text { and }(x(T), y(T))=(\bar{x}, \bar{y}) . & \end{cases}
$$

where $f_{1}(x, y, \xi)=x\left(1-\frac{x}{\gamma}\right)-\left(\frac{x y}{x+(1+\alpha \xi)\left(\omega x^{2}+1\right)}\right)$ and $f_{2}(x, y, \xi)=\beta\left(\frac{x+\xi\left(\omega x^{2}+1\right)}{x+(1+\alpha \xi)\left(\omega x^{2}+1\right)}\right) y-\delta y$. Using the equations of the system (1.5) - (1.6), we get the relation

$$
\begin{aligned}
& \qquad \begin{aligned}
f_{1}(x, y, \xi) & =(g(x, \alpha, \xi)-y) f(x, \alpha, \xi) \\
f_{2}(x, y, \xi) & =\left(\beta f(x, \alpha, \xi)\left[1+\frac{\xi}{x}\left(\omega x^{2}+1\right)\right]-\delta\right) y
\end{aligned} \\
& \text { where } f(x)=\frac{x}{(1+\alpha \xi)\left(\omega x^{2}+1\right)+x} \text { and } g(x)=\left(1-\frac{x}{\gamma}\right)\left((1+\alpha \xi)\left(\omega x^{2}+1\right)+x\right) .
\end{aligned}
$$

The control problem 4.1 is of the form of a Mayer time optimal control problem (Appendix - A) with $n=2, m=1$ and $\mathbf{x}(\mathbf{t})=(x(t), y(t)), \mathbf{u}(\mathbf{t})=\xi(t)$ with $\mathbf{f}(t, \mathbf{x}(t), \mathbf{u}(t))=\left(f_{1}(x, y, \xi), f_{2}(x, y, \xi)\right)$. The boundary conditions are given by $e[\mathbf{x}]=\left(0, x_{0}, y_{0}, T, \bar{x}, \bar{y}\right)$.

The set $A$ for this problem (4.1) is the subset of the $t \mathbf{x}$ - space $\left(\mathbb{R}^{1+2}\right)$, i.e., $A \subset \mathbb{R}^{1+2}$ which is nothing but the solution space. We define the set of all admissible solutions to the above problem is given by

$$
\Omega:=\left\{(\mathbf{x}, \xi)=(x, y, \xi):(\mathbf{x}, \xi) \text { is an admissible pair for the problem (4.1) with } \xi \in\left[\xi_{\min }, \xi_{\max }\right]\right\}
$$

\subsection{Existence of Solution for the Optimal Control Problem}

We wish to obtain a solution from the set $\Omega$ which minimizes the time to reach the terminal state $(x(T), y(T))$, that, in turn becomes the optimal solution for (4.1). We will now establish this in the following theorem by proving the existence of an optimal control using Filippov's Existence Theorem (refer to Appendix - A) which drives the system to a desired terminal state in minimum time.

Theorem 1. The optimal control problem 4.1 admits an optimal control $\xi(t)$ provided the terminal state is admissible (Propositions 1 and 2) and the the set of admissible solutions $\Omega$ is non-empty.

Proof. In order to prove this result, we will show that all the following conditions of the Filippov's Existence Theorem are satisfied by the considered control problem:

1. The set $\mathrm{A}$ is compact.

2. The set of all controls $\left[\xi_{\min }, \xi_{\max }\right]$ is compact.

3. The set of boundary points $B=\left\{0, x_{0}, y_{0}, T, \bar{x}, \bar{y}\right\}$ is compact and objective function is continuous on $B$.

4. For every $(x, y) \in A$ the sets $Q(x, y):=\left\{\left(z_{1}, z_{2}\right) \mid z_{1}=f_{1}(x, y, \xi), z_{2}=f_{2}(x, y, \xi), \xi \in\left[\xi_{\text {min }}, \xi_{\text {max }}\right]\right\}$ are convex.

We will now show that the optimal control problem (4.1) satisfies all the properties above.

(i) Whenever $\frac{\beta \xi-\delta}{\delta \xi}<0$, we know from the global dynamics of the system (Table - 2) that the solution trajectories of the system (1.3 ) - (1.4) reach $y$ - axis in finite time and are therefore closed and bounded. On the other hand, whenever $\frac{\beta \xi-\delta}{\delta \xi}>0$, we have from the positivity and boundedness theorem [45] that the solutions are closed and bounded. Thus, we can conclude that $A$ is compact and this proves condition 1. 
(ii) Conditions 2 and 3 are satisfied from the definitions of the respective sets $\left[\xi_{\min }, \xi_{\max }\right]$ and $B=$ $\left\{0, x_{0}, y_{0}, T, \bar{x}, \bar{y}\right\}$ and also by definition of the objective function $J[\xi]=T$

(iii) To prove condition 4, we need to show that the sets $Q(x, y)$ are convex. To that end, consider $z_{1}=f_{1}(x, y, \alpha)=$ $x\left(1-\frac{x}{\gamma}\right)-\left(\frac{x y}{x+(1+\alpha \xi)\left(\omega x^{2}+1\right)}\right)$. Rearranging the terms, we get

$$
\left(\frac{x y}{x+(1+\alpha \xi)\left(\omega x^{2}+1\right)}\right)=x\left(1-\frac{x}{\gamma}\right)-z_{1} .
$$

Using the fact that $z_{2}=f_{2}(x, y, \xi)=\beta\left(\frac{x+\xi\left(\omega x^{2}+1\right)}{x+(1+\alpha \xi)\left(\omega x^{2}+1\right)}\right) y-\delta y$, and from (4.2), we get

$$
z_{2}=\beta x\left(1-\frac{x}{\gamma}\right)-\beta z_{1}+\frac{\beta \xi\left(\omega x^{2}+1\right)}{x+(1+\alpha \xi)\left(\omega x^{2}+1\right)}-\delta y
$$

Now, from (4.2), we see that

$$
\xi=\frac{1}{\alpha\left(1+\omega x^{2}\right)}\left[\frac{x y}{x\left(1-\frac{x}{\gamma}\right)-z_{1}}-x-\left(\omega x^{2}+1\right)\right]
$$

and from (4.3) we have

$$
\xi\left(\omega x^{2}+1\right)\left\{\beta y-\alpha\left[\beta z_{1}+z_{2}+\beta x\left(1-\frac{1}{\gamma}\right)+\delta y\right]\right\}=\left[x+\left(\omega x^{2}+1\right)\right]\left(\beta z_{1}+z_{2}+\beta x\left(1-\frac{1}{\gamma}\right)+\delta y\right)
$$

Now, substituting for $\xi$ in the above expression (4.5) from (4.4) and simplifying the expression, we get

$$
\left(\frac{(\beta-\delta \alpha)}{\alpha}\right) y-\frac{\beta}{\alpha}\left(1-\frac{x}{\gamma}\right)\left[1+\omega x^{2}+x(1-\alpha)\right]+\frac{\beta}{\alpha x}\left[1+\omega x^{2}+x(1-\alpha)\right] z_{1}-z_{2}=0
$$

Finally, after rearranging the above expression, we get

$$
z_{2}=\frac{\beta}{\alpha x}\left[1+\omega x^{2}+x(1-\alpha)\right] z_{1}+\frac{1}{\alpha}\left[(\beta-\delta \alpha) y-\beta\left(1-\frac{x}{\gamma}\right)\left[1+\omega x^{2}+x(1-\alpha)\right]\right]
$$

The linear relation seen above (4.7) between $z_{1}$ and $z_{2}$ implies that the set $Q(x, y)$ are convex segments. This proves the condition 4.

Thus, if the set of admissible solutions to the control problem (4.1) is non-empty, i.e., $\Omega \neq \phi$, then the existence of absolute minimum can be guaranteed.

\subsection{Characteristics of the Optimal Control Solution}

In this section, we will obtain the characteristics of the optimal control solution to the control problem (4.1) assuming that the optimal control solution exists. We do this using the necessary conditions for optimal solutions given by the Pontryagin's Maximum Principle [21].

First we define the Hamiltonian function associated with the optimal control problem (4.1) as

$$
\mathbb{H}(x, y, \xi, \lambda, \mu):=\lambda \frac{d x}{d t}+\mu \frac{d y}{d t}
$$

where, $\lambda$ and $\mu$ are co-state variables or adjoint variables. We expand the above expression using the system equations (1.3) - (1.4) and we get the Hamiltonian as

$$
\mathbb{H}(x, y, \xi, \lambda, \mu)=\lambda\left[x\left(1-\frac{x}{\gamma}\right)-\left(\frac{x y}{x+(1+\alpha \xi)\left(\omega x^{2}+1\right)}\right)\right]+\mu\left[\beta\left(\frac{x+\xi\left(\omega x^{2}+1\right)}{x+(1+\alpha \xi)\left(\omega x^{2}+1\right)}\right) y-\delta y\right]
$$


By rearranging the terms, the above expression becomes

$$
\mathbb{H}(x, y, \xi, \lambda, \mu)=\left(\lambda x\left(1-\frac{x}{\gamma}\right)-\mu \delta y\right)-\frac{y}{\left(x+(1+\alpha \xi)\left(\omega x^{2}+1\right)\right)}\left(\lambda x-\mu \beta\left[x+\xi\left(\omega x^{2}+1\right)\right]\right)
$$

Also, using the symbolically modified additional food system (1.5) - (1.6) based on the definitions of the functions $f(x, \alpha, \xi)$ and $g(x, \alpha, \xi)$, we get the Hamiltonian to be

$$
\mathbb{H}(x, y, \xi, \lambda, \mu)=\lambda[(g(x, \alpha, \xi)-y) f(x, \alpha, \xi)]+\mu\left[\beta f(x, \alpha, \xi)\left(1+\frac{\xi}{x}\left(\omega x^{2}+1\right)\right)-\delta\right] y
$$

The maximum principle states that if optimal solution exists, then the co-state variables satisfy a system of equations called the Canonical equations, also called the Adjoint system, given by $\frac{d \lambda}{d t}=-\frac{\partial \mathbb{H}}{d x}, \frac{d \mu}{d t}=$ $-\frac{\partial \mathbb{H}}{d y}$. Using (4.10), the adjoint system can be expressed as

$$
\begin{aligned}
\frac{d \lambda}{d t}= & -\lambda\left\{g_{x}(x, \alpha, \xi) f(x, \alpha, \xi)+(g(x, \alpha, \xi)-y) f_{x}(x, \alpha, \xi)\right\} \\
& -\mu\left\{\beta f_{x}(x, \alpha, \xi)\left(1+\frac{\xi}{x}\left(\omega x^{2}+1\right)\right)+\frac{\beta \xi}{x^{2}} f(x, \alpha, \xi)\left(\omega x^{2}-1\right)\right\} y \\
\frac{d \mu}{d t}= & \lambda f(x, \alpha, \xi)-\mu\left\{\beta f(x, \alpha, \xi)\left(1+\frac{\xi}{x}\left(\omega x^{2}+1\right)\right)-\delta\right\}
\end{aligned}
$$

Now, we differentiate the Hamiltonian function (4.9) with respect to the control parameter $\xi$ in order to obtain the characteristics of the optimal control.

$$
\frac{\partial \mathbb{H}}{\partial \xi}=\frac{y\left(\omega x^{2}+1\right)}{\left[x+(1+\alpha \xi)\left(\omega x^{2}+1\right)\right]^{2}}\left(\lambda \alpha x+\mu \beta\left[\left(1+\omega x^{2}\right)+x(1-\alpha)\right]\right)
$$

We can observe that the optimal control $\xi^{\star}(t)$ cannot be explicitly obtained from the above relation. Thus, when we differentiate the Hamiltonian function further with respect to $\xi$, we get

$$
\frac{\partial^{2} \mathbb{H}}{\partial \xi^{2}}=-\frac{2 \alpha\left(\omega x^{2}+1\right)}{\left[x+(1+\alpha \xi)\left(\omega x^{2}+1\right)\right]} \frac{\partial \mathbb{H}}{\partial \xi}
$$

We can now conclude from the above expression (4.13) that the Hamiltonian function is a monotone with respect to the parameter $\xi$ provided the we have $\frac{\partial \mathbb{H}}{\partial \xi} \neq 0$. Using the Hamiltonian maximization condition of the maximum principle (which becomes a minimization condition in our case by the definition of the objective function) [21], we see that

$$
\mathbb{H}\left(x^{\star}(t), y^{\star}(t), \xi^{\star}(t), \lambda^{\star}(t), \mu^{\star}(t)\right) \leq \mathbb{H}\left(x^{\star}(t), y^{\star}(t), \xi, \lambda^{\star}(t), \mu^{\star}(t)\right)
$$

$\forall \xi \in\left[\xi_{\text {min }}, \xi_{\text {max }}\right]$ and $\forall t \in[0, T]$. Also, since the formulated control problem (4.1) is a time optimal control problem, the Hamiltonian function would turn out being a constant along the optimal trajectory and in particular, it assumes value -1 [9]. Hence,

$$
\mathbb{H}(x(t), y(t), \alpha, \lambda(t), \mu(t))=-1
$$

Thus, using equation (4.9), the condition (4.14), and the monotonicity property of Hamiltonian function with respect to $\xi$, we conclude that the optimal control solution $\xi^{\star}(t)$ could be of bang-bang type if no singularity exists in any sub-interval of $0, T$. This implies that optimal control function would assume the form: 


$$
\xi^{*}(t)=\left\{\begin{array}{lll}
\xi_{\max }, & \text { if } & \frac{\partial \mathbb{H}}{\partial \xi}<0 \\
\xi_{\min }, & \text { if } & \frac{\partial \mathbb{H}}{\partial \xi}>0 \\
? & \text { if } & \frac{\partial \mathbb{H}}{\partial \xi}=0
\end{array}\right.
$$

As mentioned above, singularity in optimal solution occurs when $\frac{\partial \mathbb{H}}{\partial \xi}=0$ for a sub-interval of $[0, T]$. In this situation, the optimal control function can no longer be obtained using the Hamiltonian minimization condition and the monotonicity property. To show that the optimal solution is of bang-bang type only, we must rule out the existence of a singular arc in any interval $\left(t_{1}, t_{2}\right), \subseteq[0, T]$. Thus, to precisely the characterize the optimal control, we first assume that singular solution exists and then show that singular arc actually does not exist.

Let $\frac{\partial \mathbb{H}}{\partial \xi}=0$ for some time instant $t \in[0, T]$. This implies that

$$
\lambda \alpha x+\mu \beta\left[\left(1+\omega x^{2}\right)+x(1-\alpha)\right]=0
$$

Rearranging the terms in the above equation, we get

$$
\frac{\lambda}{\mu}=\frac{\beta\left[x(\alpha-1)-\left(\omega x^{2}+1\right)\right]}{\alpha x}
$$

From the above equation we can infer that along the singular solution, the co-state variables behave as follows:

(i) $\lambda$ and $\mu$ will be of opposite signs when $\omega x^{2}-(\alpha-1) x+1>0$.

(ii) $\lambda$ and $\mu$ will be of same sign when $\omega x^{2}-(\alpha-1) x+1<0$.

(iii) $\lambda=0$ and $\mu$ will be arbitrary when $\omega x^{2}-(\alpha-1) x+1=0$

The behaviour of the co-state variables along the singular solution shows that both of them cannot become zero simultaneously because that would lead to a contradiction with respect to the Hamiltonian along the optimal trajectory given by equation (4.15). Thus, to characterize the optimal trajectory along the singular solution, we differentiate the equation (4.11) with respect to time. This gives us

$$
\begin{aligned}
\frac{d}{d t} \frac{\partial \mathbb{H}}{\partial \xi} & =\frac{d}{d t}\left[\frac{y\left(\omega x^{2}+1\right)}{\left[x+(1+\alpha \xi)\left(\omega x^{2}+1\right)\right]^{2}}\left(\lambda \alpha x+\mu \beta\left[\left(1+\omega x^{2}\right)+x(1-\alpha)\right]\right)\right]=0 \\
& =\left(\lambda \alpha x+\mu \beta\left[1+\omega x^{2}+x(1-\alpha)\right]\right) \frac{d}{d t}\left(\frac{y\left(\omega x^{2}+1\right)}{\left[x+(1+\alpha \xi)\left(\omega x^{2}+1\right)\right]^{2}}\right) \\
& +\left(\frac{y\left(\omega x^{2}+1\right)}{\left[x+(1+\alpha \xi)\left(\omega x^{2}+1\right)\right]^{2}}\right) \frac{d}{d t}\left(\lambda \alpha x+\mu \beta\left[1+\omega x^{2}+x(1-\alpha)\right]\right)=0
\end{aligned}
$$

Using the fact that equation (4.16) holds along singular solution, the above expression becomes

$$
\frac{d}{d t} \frac{\partial \mathbb{H}}{\partial \xi}=\left(\frac{y\left(\omega x^{2}+1\right)}{\left[x+(1+\alpha \xi)\left(\omega x^{2}+1\right)\right]^{2}}\right) \frac{d}{d t}\left(\lambda \alpha x+\mu \beta\left[1+\omega x^{2}+x(1-\alpha)\right]\right)=0
$$

Expanding the above expression on the right hand side, we get

$$
\frac{d}{d t}\left(\lambda \alpha x+\mu \beta\left[1+\omega x^{2}+x(1-\alpha)\right]\right)=\alpha x \frac{d \lambda}{d t}+\beta\left[1+\omega x^{2}+(1-\alpha) x\right] \frac{d \mu}{d t}+[\lambda \alpha+\mu \beta(2 \omega x+(1-\alpha))] \frac{d x}{d t}
$$


Expanding the terms in the above expression using the additional food system (1.5) - (1.6), the adjoint system (4.11) and simplifying the terms using the condition (4.16) which is satisfied along the singular solution, the equation (4.19) becomes

$$
\frac{d}{d t}\left(\lambda \alpha x+\mu \beta\left[1+\omega x^{2}+\chi(1-\alpha)\right]\right)=-\frac{\mu \beta}{\gamma \alpha}\left[3 \omega \gamma x^{3}+[\gamma \omega[\beta-\alpha \delta+2 \alpha]+\alpha(1-\alpha)] x^{2}-[\gamma(1-\alpha)(\beta-\delta \alpha+\alpha)-\alpha] x+\gamma(\beta-\delta \alpha)\right]
$$

Substituting (4.20) in (4.18), we get

$$
\begin{aligned}
\frac{d}{d t} \frac{\partial \mathbb{H}}{\partial \xi}= & \left(\frac{\mu \beta y\left(\omega x^{2}+1\right)}{\gamma \alpha\left[x+(1+\alpha \xi)\left(\omega x^{2}+1\right)\right]^{2}}\right)\left[-3 \omega \gamma x^{3}-[\gamma \omega(\beta-\alpha(\delta-2))-2 \alpha(\alpha-1)] x^{2}\right. \\
& +[\gamma(\alpha-1)(\beta-\alpha(\delta+1))-\alpha] x-\gamma(\beta-\delta \alpha)]=0
\end{aligned}
$$

The above equation implies that along the singular solution, we have

$$
3 \omega \gamma x^{3}+[\gamma \omega(\beta-\alpha(\delta-2))-2 \alpha(\alpha-1)] x^{2}-[\gamma(\alpha-1)(\beta-\alpha(\delta+1))-\alpha] x+\gamma(\beta-\delta \alpha)=0
$$

The above relation implies that if singularity occurs in the optimal solution of the control problem (4.1), then it does at the roots of the above cubic equation (provided the roots are real and positive). To get more insight into the points of singularity, we differentiate equation (4.21) again with respect to time along singular solution assuming that at least one root of the cubic equation (4.22) is real and positive (denoted by $\hat{x}$ ). Then, using equation (4.16) we get

$$
\begin{aligned}
\frac{d^{2}}{d t^{2}} \frac{\partial \mathbb{H}}{\partial \xi}= & \left(\frac{\mu \beta y\left(\omega x^{2}+1\right)}{\gamma \alpha\left[x+(1+\alpha \xi)\left(\omega x^{2}+1\right)\right]^{2}}\right)\left[-9 \omega \gamma \hat{x}^{2}-2[\gamma \omega(\beta-\alpha(\delta-2))-2 \alpha(\alpha-1)] \hat{x}\right. \\
& +[\gamma(\alpha-1)(\beta-\alpha(\delta+1)-\alpha)](g(\hat{x}, \alpha, \xi)-y) f(\hat{x}, \alpha, \xi)=0
\end{aligned}
$$

from which we can conclude that if singularity occurs in the optimal control solution of the control problem (4.1) and if at least one of the roots of the cubic equation (4.22) denoted by $\hat{x}$ is real and positive, then the $y$ component of the point in the solution space where singularity occurs is given by

$$
\hat{y}=g(\hat{x}, \alpha, \xi)
$$

Thus, we conclude that if singularity occurs, it will be at points $(\hat{x}, \hat{y})$ and there is no possibility for a singular arc. Hence, we note that that the optimal control can be established using the Hamiltonian minimization condition and monotonicity property as guessed above. Summarizing the above analysis we will now state a result that characterizes the optimal solution of the control problem (4.1)

Theorem 2. The optimal control strategy for the time optimal control problem (4.1) is a combination of bangbang controls only, with possibility of switches occurring at points in the optimal trajectory. The Optimal control is given by

$$
\xi^{\star}(t)=\left\{\begin{array}{lll}
\xi_{\max }, & \text { if } & \frac{\partial \mathbb{H}}{\partial \xi}<0 \\
\xi_{\min }, & \text { if } & \frac{\partial \mathbb{H}}{\partial \xi}>0
\end{array}\right.
$$

Now, based on the theorem stated above, we state a corollary to the existence theorem (Theorem 1).

Corollary 1. If there exists an admissible path connecting the initial state $\left(x_{0}, y_{0}\right)$ and the terminal state $(\bar{x}, \bar{y})$ involving a combination of bang-bang controls, then the optimal control problem (4.1) has an optimal solution. 
In the previous section, we saw that if the system admits both equilibria, then the desired terminal state may not be admissible (or cannot be reached) if a homoclinic orbit exists. However, now we see from (4.25) that the optimal control strategy is a bang-bang strategy that alternates between extremes. Also, since the existence of homoclinc orbits depends on the choice of $\xi^{\star}$ for a constant $\alpha$, we see that if for one of the extreme values $\xi_{\min }$ or $\xi_{\max }$, the homoclinic orbit does not exist, then we can still drive the system to the desired terminal state. We will depict this scenario though a numerical simulations in the later section.

\section{Optimal Solution Trajectories and Applications to Pest Management}

In this, section, we study the nature of the optimal solution trajectories and approach paths based on the switch points (if singularity occurs) and analyse the optimal solution. We will specifically see the applications of these results in the context of pest management. For that, we first consider the following equation whose roots give the points of singularity in the optimal solution

$$
F(x) \equiv 3 \omega \gamma x^{3}+[\gamma \omega(\beta-\alpha(\delta-2))-2 \alpha(\alpha-1)] x^{2}-[\gamma(\alpha-1)(\beta-\alpha(\delta+1))-\alpha] x+\gamma(\beta-\delta \alpha)=0
$$

In order to know about the existence and nature of the singular points, we need to establish the existence of number of roots of the above equation (5.1). Using the Descarte's rule of signs and thereby understanding the co-efficients and the parameters involved, we conclude that the the cubic equation (5.1) can have either one, two or no real roots. Accordingly, the phase space can be divided into various regions as follows:

1. Case I: $F(x)$ has no positive roots: In this case, we can divide the phase space into two regions as follows:

$$
\begin{aligned}
& \text { Region Ia }:=\left\{(x, y) \mid y<\frac{\beta}{\beta-\delta \alpha}\left(1-\frac{x^{\star}(\xi)}{\gamma}\right)\left(1+\omega\left(x^{\star}(\xi)\right)^{2}+x^{\star}(\xi)(1-\alpha)\right)\right\} \\
& \text { Region Ib }:=\left\{(x, y) \mid y>\frac{\beta}{\beta-\delta \alpha}\left(1-\frac{x^{\star}(\xi)}{\gamma}\right)\left(1+\omega\left(x^{\star}(\xi)\right)^{2}+x^{\star}(\xi)(1-\alpha)\right)\right\}
\end{aligned}
$$

2. Case II: $F(x)$ has one positive root denoted by $\hat{x}$. In this case, we can divide the phase space into four regions as follows:

$$
\begin{aligned}
& \text { Region IIa }:=\left\{(x, y) \mid y<\frac{\beta}{\beta-\delta \alpha}\left(1-\frac{x^{\star}(\xi)}{\gamma}\right)\left(1+\omega\left(x^{\star}(\xi)\right)^{2}+x^{\star}(\xi)(1-\alpha)\right) \text { and } x<\hat{x}\right\} \\
& \text { Region IIb }:=\left\{(x, y) \mid y<\frac{\beta}{\beta-\delta \alpha}\left(1-\frac{x^{\star}(\xi)}{\gamma}\right)\left(1+\omega\left(x^{\star}(\xi)\right)^{2}+x^{\star}(\xi)(1-\alpha)\right) \text { and } x>\hat{x}\right\} \\
& \text { Region IIc }:=\left\{(x, y) \mid y>\frac{\beta}{\beta-\delta \alpha}\left(1-\frac{x^{\star}(\xi)}{\gamma}\right)\left(1+\omega\left(x^{\star}(\xi)\right)^{2}+x^{\star}(\xi)(1-\alpha)\right) \text { and } x>\hat{x}\right\} \\
& \text { Region IId }:=\left\{(x, y) \mid y>\frac{\beta}{\beta-\delta \alpha}\left(1-\frac{x^{\star}(\xi)}{\gamma}\right)\left(1+\omega\left(x^{\star}(\xi)\right)^{2}+x^{\star}(\xi)(1-\alpha)\right) \text { and } x<\hat{x}\right\}
\end{aligned}
$$


3. Case III: $F(x)$ has two positive roots denoted by $\bar{x}$ and $\hat{x}$. Let us assume without any loss in generality that $\bar{x}<\hat{x}$. In this case, we can divide the phase space into five regions as follows:

$$
\begin{aligned}
& \text { Region IIIa }:=\left\{(x, y) \mid y<\frac{\beta}{\beta-\delta \alpha}\left(1-\frac{x^{\star}(\xi)}{\gamma}\right)\left(1+\omega\left(x^{\star}(\xi)\right)^{2}+x^{\star}(\xi)(1-\alpha)\right) \text { and } \bar{x}<x<\hat{x}\right\} \\
& \text { Region IIIb }:=\left\{(x, y) \mid y<\frac{\beta}{\beta-\delta \alpha}\left(1-\frac{x^{\star}(\xi)}{\gamma}\right)\left(1+\omega\left(x^{\star}(\xi)\right)^{2}+x^{\star}(\xi)(1-\alpha)\right) \text { and } \bar{x}<\hat{x}<x\right\} \\
& \text { Region IIIc }:=\left\{(x, y) \mid y>\frac{\beta}{\beta-\delta \alpha}\left(1-\frac{x^{\star}(\xi)}{\gamma}\right)\left(1+\omega\left(x^{\star}(\xi)\right)^{2}+x^{\star}(\xi)(1-\alpha)\right) \text { and } x<\bar{x}<\hat{x}\right\} \\
& \text { Region IIId }:=\left\{(x, y) \mid y>\frac{\beta}{\beta-\delta \alpha}\left(1-\frac{x^{\star}(\xi)}{\gamma}\right)\left(1+\omega\left(x^{\star}(\xi)\right)^{2}+x^{\star}(\xi)(1-\alpha)\right) \text { and } \bar{x}<x<\hat{x}\right\} \\
& \text { Region IIIe }:=\left\{(x, y) \mid y>\frac{\beta}{\beta-\delta \alpha}\left(1-\frac{x^{\star}(\xi)}{\gamma}\right)\left(1+\omega\left(x^{\star}(\xi)\right)^{2}+x^{\star}(\xi)(1-\alpha)\right) \text { and } \bar{x}<\hat{x}<x\right\}
\end{aligned}
$$

Now, we will state and prove a result which describes the nature of switching (if it occurs), depending on the regions that divide the phase plane.

Proposition 3. The optimal control $\xi^{\star}(t)$ along the optimal trajectory switches from $\xi_{\min }$ to $\xi_{\max }$ (or $\xi_{\max }$ to $\xi_{\text {min }}$ ) in Regions Ia, IIa, IId, IIIb and IIId (or in Regions Ib, IIb, IIc, IIIa, IIIc and IIIe) only.

Proof. We know from the results of the previous section that when a switch occurs along the optimal trajectory, then at that instant we have $\frac{\partial \mathbb{H}}{\partial \xi}=0$. From (4.12), we get

$$
\lambda(t) \alpha(x(t))+\beta \mu(t)\left[1+\omega(x(t))^{2}+x(t)(1-\alpha)\right]=0
$$

Let us denote $t=\tau$ as the time instant at which switch occurs. Then we get

$$
\lambda(\tau) \alpha(x(\tau))+\beta \mu(\tau)\left[1+\omega(x(\tau))^{2}+x(\tau)(1-\alpha)\right]=0
$$

Since (4.15) holds along the optimal trajectory, we get

$$
\mathbb{H}(x(\tau), y(\tau), \xi(\tau), \lambda(\tau), \mu(\tau))=-1
$$

Using the definition of Hamiltonian from equation (4.9), we get

$$
\begin{aligned}
\left(\lambda(\tau) x(\tau)\left(1-\frac{x(\tau)}{\gamma}\right)-\mu(\tau) \delta y(\tau)\right) & -\frac{y(\tau)}{\left[x+\left(1+(x(\tau))^{2}\right)(1+\alpha \xi(\tau))\right]} \\
& \left(\lambda(\tau)(x(\tau))-\mu(\tau) \beta\left((x(\tau))+\xi(\tau)\left(1+\omega(x(\tau))^{2}\right)\right)\right)=-1
\end{aligned}
$$

From equation (5.2), we get

$$
\lambda(\tau) x(\tau)=-\frac{\beta}{\alpha} \mu(\tau)\left[1+\omega(x(\tau))^{2}+x(\tau)(1-\alpha)\right]
$$

Now, substituting (5.4) in (5.3) followed by some simplification of the terms, we get

$$
\lambda(\tau) x(\tau)\left(1-\frac{x(\tau)}{\gamma}\right)-\mu(\tau) \delta y(\tau)+\frac{\beta \mu(\tau) y(\tau)}{\alpha}=-1
$$

Since we arrived at the condition that $\mu \neq 0$ along the optimal solution, multiplying both sides of the above equation by $\frac{\alpha}{\mu(\tau)}$ and using the expression for $\frac{\lambda(\tau)}{\mu(\tau)}$ along optimal solution, we get

$$
\frac{\beta\left[(x(\tau))(\alpha-1)-\left(1+\omega(x(\tau))^{2}\right)\right]}{x(\tau)}\left(1-\frac{x(\tau)}{\gamma}\right)+(\beta-\delta \alpha) y(\tau)=-\frac{\alpha}{\mu(\tau)}
$$


After rearranging the above expression, we get

$$
\mu(\tau)=-\frac{\alpha}{(\beta-\delta \alpha)\left[y(\tau)-\frac{\beta}{\beta-\delta \alpha}\left(1-\frac{\chi(\tau)}{\gamma}\right)\left[1+\omega(x(\tau))^{2}+x(\tau)(1-\alpha)\right]\right]}
$$

From the nature of the curve (3.4) and the definition of various regions, we see that $\mu(\tau)$ is positive (negative) in the regions Ia, II a, IId, III a, IIIb and IIId (Ib, IIIb, IIC, IIIa, IIIC, and IIIe).

Now, let $\sigma(t)=\lambda(t) \alpha(x(t))+\beta \mu(t)\left[1+\omega(x(t))^{2}+x(t)(1-\alpha)\right]$ and consider

$$
\frac{d \sigma}{d t}=\frac{d}{d t}\left(\lambda(t) \alpha(x(t))+\beta \mu(t)\left[1+\omega(x(t))^{2}+x(t)(1-\alpha)\right]\right)
$$

Using the equations (4.19), (4.20) and using the fact that $\sigma(\tau)=0$, we get

$$
\left.\frac{d \sigma}{d t}\right|_{t=\tau}=\left.\frac{\mu \beta}{\gamma \alpha}\left[3 \omega \gamma x^{3}+[\gamma \omega(\beta-\alpha(\delta-2))-2 \alpha(\alpha-1)] x^{2}-[\gamma(\alpha-1)(\beta-\alpha(\delta+1))-\alpha] x+\gamma(\beta-\delta \alpha)\right]\right|_{t=\tau}
$$

From the optimal control strategy (4.25), we see that when the control switches from $\xi_{\max }$ to $\xi_{\min }$ (or $\xi_{\min }$ to $\xi_{\max }$ ) at $t=\tau$, then $\frac{\partial \mathbb{H}}{\partial \xi}$ accordingly increases from negative to positive (positive to negative). Thus, $\frac{d \sigma}{d t}>0(<0)$ at $t=\tau$ for the switch $\xi_{\max }$ to $\xi_{\min }$ (or $\xi_{\min }$ to $\xi_{\max }$ ). Using observations along with the equations (5.6) and (5.7) we can conclude that the switch $\xi_{\max }$ to $\xi_{\min }\left(\xi_{\min }\right.$ to $\xi_{\max }$ ) can occur in Ia, IIa, IId, IIIa, IIIb and IIId (Ib, IIb , IIC, III a, IIIC, and IIIe) only.

The above result (Proposition 3) shows that for reaching an interior point in the solution space, the optimal control may involve switching between values $\left(\xi_{\min }\right)$ and $\left(\xi_{\max }\right)$ at multiple points. This means that in order to achieve biological conservation, the additional food supply could involve switches in the amount of food that is supplied so that co-existence of species is achieved. Now, we will focus on the relevance of the mathematical analysis hitherto in the case of pest management.

From the existence theorem (Theorem 1), we see that in the context of pest management, in order to reach the desired state at finite time, our goal is to reach the terminal prey density as $x(T)=0$. Now, considering the desired terminal state to be $(0, y(T))$, we state two important results that establish the characteristics of the co-state variables at the terminal time $t=T$ and the characteristics of the optimal control throughout the optimal trajectory leading to pest eradication.

Lemma 1. Let $\beta-\delta \alpha>0$ and $x(T)=0$. Then the time optimal control problem (4.1) admits an optimal solution if $\xi_{\max }>\frac{\delta}{\beta-\delta \alpha}$. Moreover, if $0>\lambda(T)>\frac{-1}{\epsilon}$ at the final time $t=T$, then $\xi^{\star}(T)=\xi_{\text {opt }}(T)=\xi_{\text {max }}$ with $\mu(T)<0$.

Proof. Let $\xi_{\max }>\frac{\delta}{\beta-\delta \alpha}$. Then, we can deduce from the analysis presented in the table - 2 that the system leads to pest eradication (prey elimination). With $x(T)=0$, we see that $y(T)=y(0)$ touches the predator axis in finite time due to the nature of solution trajectories and the positivity and boundedness of the solutions. Hence, for the control problem (4.1), $\Omega \neq \phi$. Hence, by using the Existence theorem (Theorem 1), we conclude that there exists an optimal solution to the control problem (4.1).

Now, since the terminal state is $\left(0, y^{\star}(T)\right)$, according to our earlier analysis, we must have $\xi^{\star}(T)>\frac{\delta}{\beta-\delta \alpha}$. Using this and the equation (4.15) along the optimal trajectory, at the terminal state, we have

$$
\begin{aligned}
\mu(T)\left(\frac{\beta \xi^{\star}(T)}{1+\alpha \xi^{\star}(T)}-\delta\right) y(T) & =-1 \\
\mu(T)\left(\frac{\beta \xi^{\star}(T)}{1+\alpha \xi^{\star}(T)}-\delta\right) & =\frac{-1}{y(T)}
\end{aligned}
$$

Rearranging the terms above, we get

$$
\mu(T)=-\frac{1}{\left(\frac{\beta \xi^{\star}(T)}{1+\alpha \xi^{\star}(T)}-\delta\right) y^{\star}(0)}
$$


Since $\xi^{\star}(T)>\frac{\delta}{\beta-\delta \alpha}$, we have $\frac{\beta \xi^{\star}(T)}{1+\alpha \xi^{\star}(T)}-\delta>0$ implying that $\mu(T)<0$ which implies that

$$
\left.\frac{\partial \mathbb{H}}{\partial \xi}\right|_{t=T}=\left(\frac{y(T)}{\left(1+\alpha \xi^{\star}(T)\right)^{2}}\right)(\mu(T) \beta)<0
$$

Therefore, from Theorem 2, we get that $\xi_{\text {opt }}(T)=\xi^{\star}(T)=\xi_{\text {max }}$. This proves the lemma.

Theorem 3. If $\xi_{\max }>\frac{\delta}{\beta-\delta \alpha}$, then the solution to the optimal control problem (4.1) with the terminal state $(x(T), y(T))=\left(0, y^{\star}(0)\right)$ is given by $\xi_{\text {opt }}(t)=\xi^{\star}(t)=\xi_{\max } \forall t \in[0, T]$.

Proof. We will use the zero solution of the linear system of the co-state variables (4.11) along the optimal path to prove this theorem. The matrix form of the system (4.11) is given by

$$
\left(\begin{array}{l}
\frac{d \lambda}{d t} \\
\frac{d \mu}{d t}
\end{array}\right)=\left(\begin{array}{ll}
-a_{1}(t) & -b_{1}(t) \\
a_{2}(t) & -b_{2}(t)
\end{array}\right)\left(\begin{array}{l}
\lambda(t) \\
\mu(t)
\end{array}\right)
$$

where

$$
\begin{aligned}
& a_{1}(t)=1-\frac{2 x}{\gamma}-\frac{(1+\alpha \xi)\left(1-\omega x^{2}\right) y}{\left[x+(1+\alpha \xi)\left(\omega x^{2}+1\right)\right]^{2}} \\
& b_{1}(t)=\frac{\beta y(1+\alpha \xi-\xi)\left(\omega x^{2}+1\right)}{\left[x+(1+\alpha \xi)\left(\omega x^{2}+1\right)\right]^{2}} \\
& a_{2}(t)=\frac{x}{\left[x+(1+\alpha \xi)\left(\omega x^{2}+1\right)\right]} \\
& b_{2}(t)=\frac{\beta\left(x+\xi\left(\omega x^{2}+1\right)\right)}{\left[x+(1+\alpha \xi)\left(\omega x^{2}+1\right)\right]}-\delta
\end{aligned}
$$

From the expansion of the matrix co-efficients given above, we observe that

- $a_{2}(t)>0$

- The sign of $b_{1}(t)$ can be determined based on the sign of the term $1+\alpha \xi-\xi$.

- If $\xi(t)=\xi_{\max }$, then $b_{2}(t)>0$ by the hypothesis of the theorem

- $a_{1}(t)$ can either be negative or positive given the values of the state variables and parameters

The characteristic equation of the system (5.9) is given by

$$
m^{2}+\left(a_{1}(t)+b_{2}(t)\right) m+\left(a_{1}(t) b_{2}(t)+a_{2}(t) b_{1}(t)\right)=0
$$

We know that based on the properties of the functions $\left(a_{1}(t)+b_{2}(t)\right)$ and $\left(a_{1}(t) b_{2}(t)+a_{2}(t) b_{1}(t)\right)$ used in the characteristic equation (5.10) we can understand the qualitative properties of the solution of the system (5.9). Assuming that $\lambda(T)<0$, using the continuity of functions $\left(a_{1}(t)+b_{2}(t)\right)$ and $\left(a_{1}(t) b_{2}(t)+a_{2}(t) b_{1}(t)\right)$, we can imply that there exists a left neighbourhood of $T$ in the interval $[0, T]$, say $[s, T]$, such that $\lambda(t)<0$ and $\mu(T)<0$ for all $t \in[s, T]$. As a result, we have $\frac{\partial \mathbb{H}}{\partial \xi}<0$ and consequently $\xi_{\text {opt }}(t)=\xi_{\text {max }}$. The proof of this theorem would be complete if we can show that $s=0$.

Now, using the qualitative behaviour of the zero solution of the system (5.9), we will show that the initial values for $\lambda$ and $\mu$ can be chosen in such a way that $\lambda(t)<0$ and $\mu(t)<0$ for all $t \in[0, T]$ thereby proving this theorem. Let us consider two cases based on the sign of the function $b_{1}(t)$.

Case 1: $1+\alpha \xi-\xi \leq 0$

In this case, the function $b_{1}(t) \leq 0$ in the system (5.9) and the discriminant of the characteristic equation (5.10) turns out to be positive for all $t \in[0, T]$. This implies that any solution that starts in the third quadrant of the $\lambda \mu$ - space with initial values chosen such that $\lambda(0)$ and $\mu(0)$ both are negative remain in the same 
quadrant and do not move to any other quadrant. Thus makes the sign of the switching function not change throughout in $[0, T]$.

Case 2: $1+\alpha \xi-\xi>0$

In this case, $b_{1}(t)>0$ and this fact is not sufficient to conclude anything on the sign of the discriminant of the characteristic equation (5.10). Thus, there is a possibility that the solution of the system (5.9) that begins in the third quadrant of the $\lambda \mu$ - space drifts to another quadrant with time. From the prey-predator isoclines causing the interior equilibrium, we can observe that at time $t=T$, for $x(T)=0$, we have $y(T)>1+\alpha \xi_{\text {max }}$. This means that the zero solution of the system (5.9) behaves as a saddle when time $t$ is closer to the terminal time $T$. Thus, in order to ensure that the solution of the system (5.9) remains in the third quadrant, the initial values must be chosen such that $\mu(0)$ is far away from zero on the negative $\mu$ - axis and $\lambda<0$, so that when the co-state solution nears negative $\lambda$ - axis as $t \rightarrow T$, then the saddle nature of the zero solution prevents it from going out of the third quadrant in the $\lambda \mu$ - space.

Thus we have $\xi_{\text {opt }}(t)=\xi_{\max }$ for all $t \in[0, T]$ in both the cases. This proves the theorem.
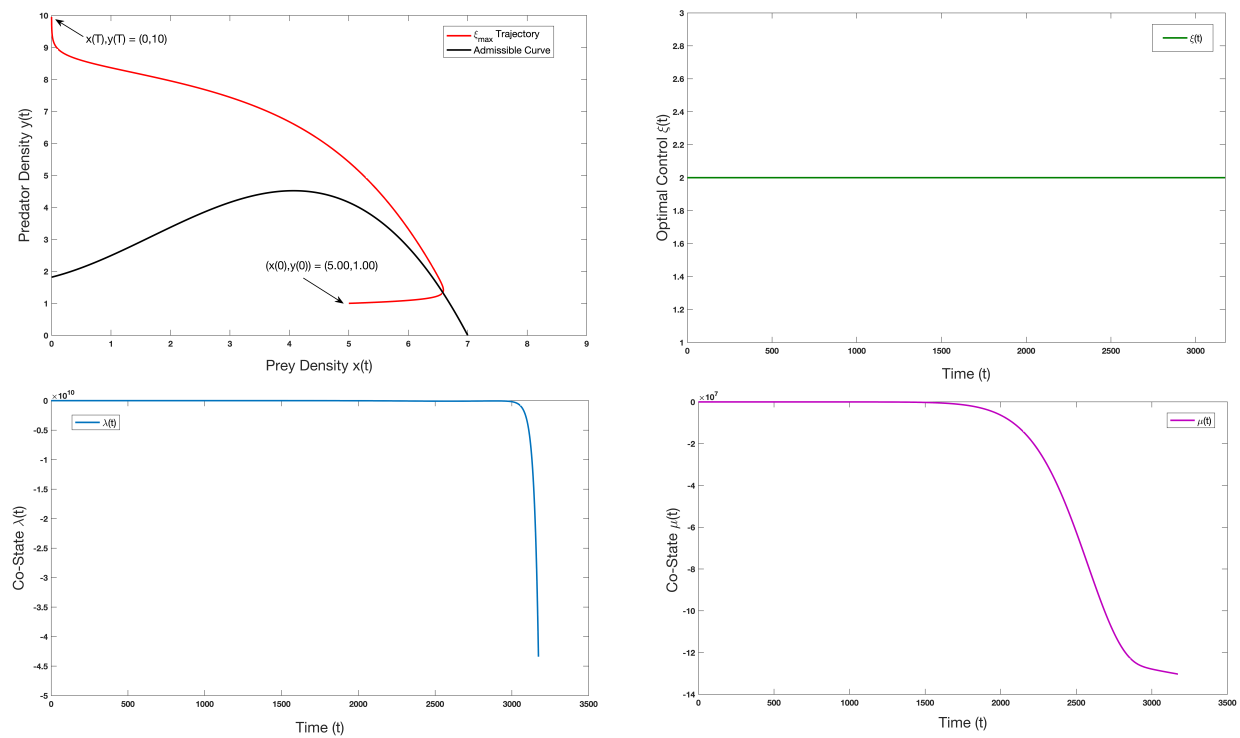

Figure 9: This figure depicts the optimal trajectory of the time optimal control problem (4.1) from the initial state (5.00, 1.00) to the terminal state $(0,10)$ with the parameters values $\gamma=7 \beta=0.4, \delta=0.3, \alpha=0.6, \omega=0.2, \xi_{\min }=1$, and $\xi_{\max }=2$. The initial value chosen for co-state variables is $(\lambda(0), \mu(0))=(-8,-4)$. Based on the table - 2 , for this example $S=1.36$ and accordingly $S<\xi_{\max }$. Based on our earlier analysis, the solution trajectories should lead to prey elimination. This example shows how the results obtained can be applied in case of pest management. This example illustrates Theorem 3 where the optimal control does not undergo any switch. Also, the co-state variables are negative throughout the optimal trajectory. This examples shows that when high quality additional food of maximum quantity is provided to the predators, then prey (pest) eradicated from the ecosystem. The desired terminal state is reached in $T=31.73$ units of time.

\section{Numerical Illustrations}

In this section, we present numerical examples that illustrate the results established in the previous section. We have performed the simulations on MATLAB software. In order to obtain the optimal solution, first 
we fixed the initial and terminal states and accordingly the range of the control parameter $\xi(t)$ as $\left[\xi_{\min }, \xi_{\max }\right]$. Using the fact that the Hamiltonian function is constant with value -1 along the optimal trajectory, we fix the initial values of co-state vectors with various combinations of trial and error processes with the goal of reaching the terminal state. We then simulate the systems (1.3) - (1.4) and (4.11) using the 4th order RungeKutta routines and switched the control using the switching function $\frac{\partial \mathbb{H}}{\partial \xi}$ while monitoring the Hamiltonian function until the terminal state was reached. We used the step size $h=0.01$ for the simulations, based on which the time units obtained at the end of simulations are accordingly re-scaled by multiplying $T \times 10^{-2}$.
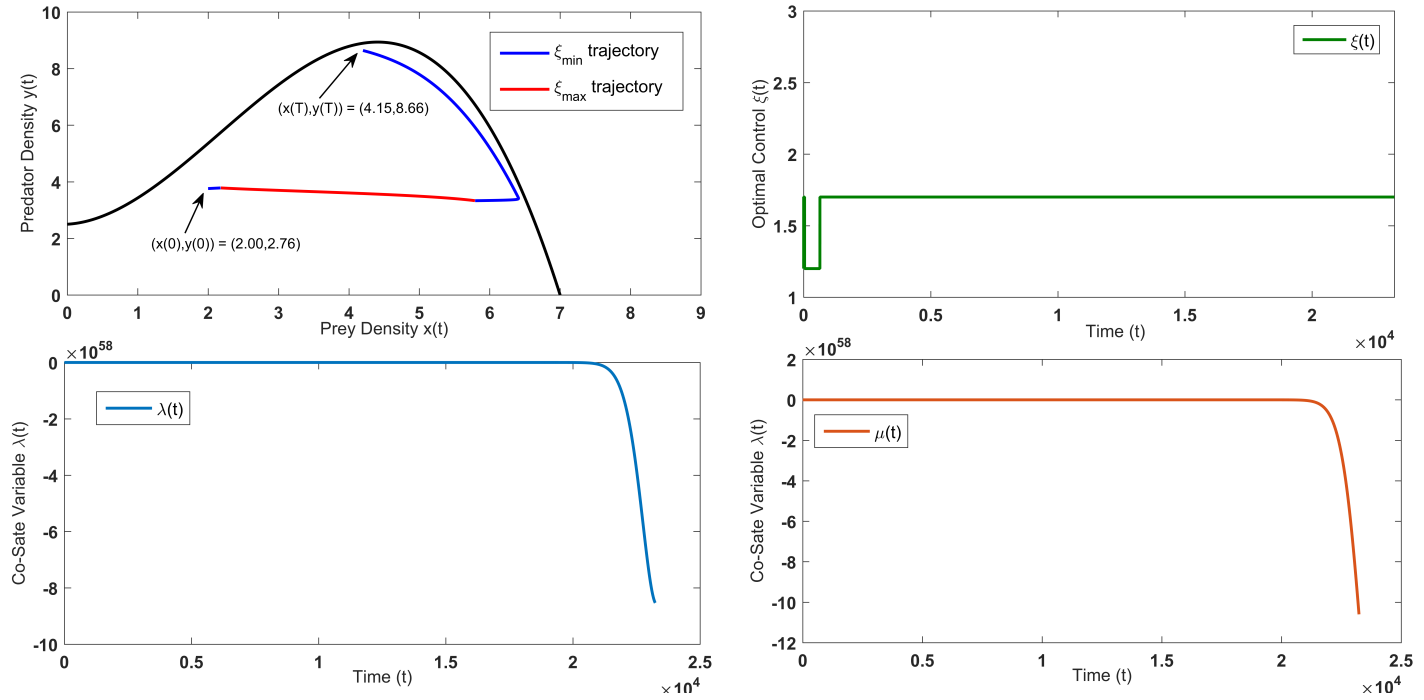

Figure 10: This figure depicts the optimal trajectory of the time optimal control problem (4.1) from the initial state (2.00, 2.76) to the terminal state $(4.15,8.66)$ with the parameters values $\gamma=7 \beta=0.4, \delta=0.3, \alpha=0.8, \omega=0.4, \xi_{\text {min }}=$ 1.2 , and $\xi_{\max }=1.7$. The initial value chosen for co-state variables is $(\lambda(0), \mu(0))=(-3,3.35)$. Based on the table -2 , for this example we have $P=1.38, Q=1.66$ and $S=1.875$ and accordingly $\xi_{\min }<P<Q<\xi_{\max }<S$. We see here that this situation is an example of Case la where there exists an asymptotically stable limit cycle around the unstable interior equilibrium. The desired terminal state is reached in $T=312.93$ units of time. This is an example where both the prey and predator densities are increased in the process of providing additional food of appropriate quantity. In particular, the predator density is significantly increased by predominantly providing additional food of high quantity. We observe that the optimal control switches twice depending on the switching function. This example depicts the case where multiple switches are involved to bring in co-existence of species illustrating the findings Theorem 2. 

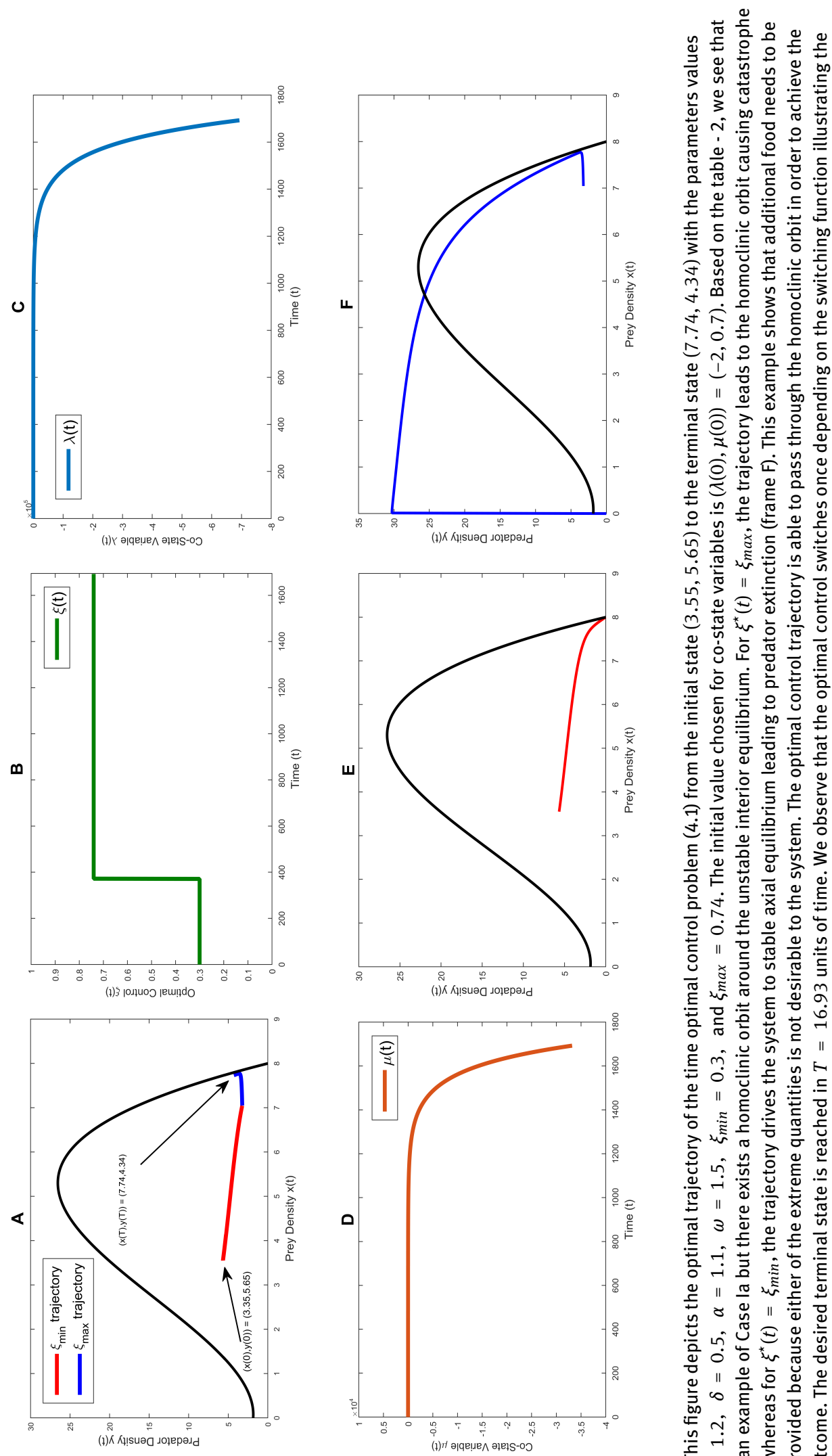

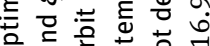

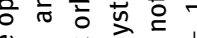

.

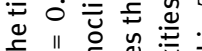

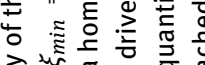

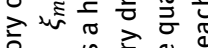

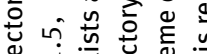

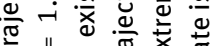

$\frac{\pi}{\pi} 3$ 过

E

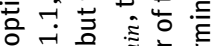

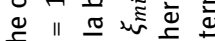

艺

.

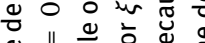

๑

志

告

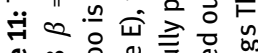

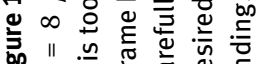

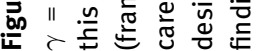



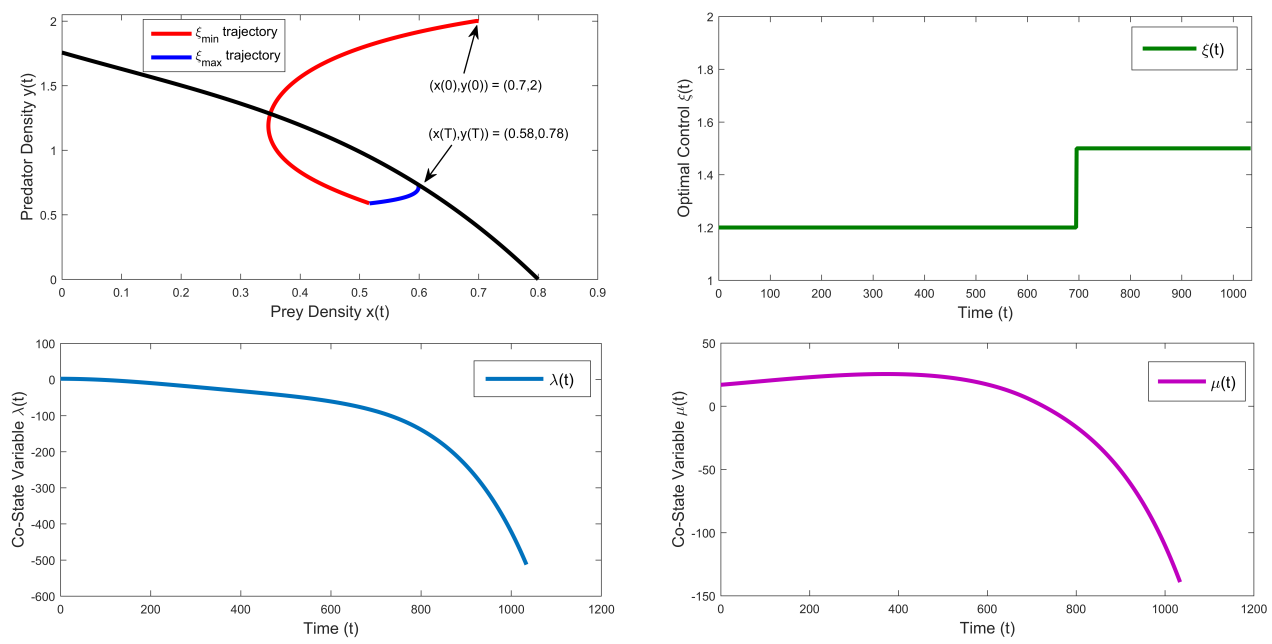

Figure 12: This figure depicts the optimal trajectory of the time optimal control problem (4.1) from the initial state $(0.7,2)$ to the terminal state $(0.58,0.78)$ with the parameters values $\gamma=0.8 \beta=2.67, \delta=2.3, \alpha=0.5, \omega=1, \xi_{\min }=1.2$, and $\xi_{\text {max }}=$ 1.5. The initial value chosen for co-state variables is $(\lambda(0), \mu(0))=(2,17)$. This is an example where the predator density is significantly brought down by providing low quantity of additional food initially and then increasing the quantity to sustain there and ensure co-existence. The desired terminal state is reached in $T=10.34$ units of time. We observe that the optimal control switches once depending on the switching function illustrating the findings of Theorem 2.
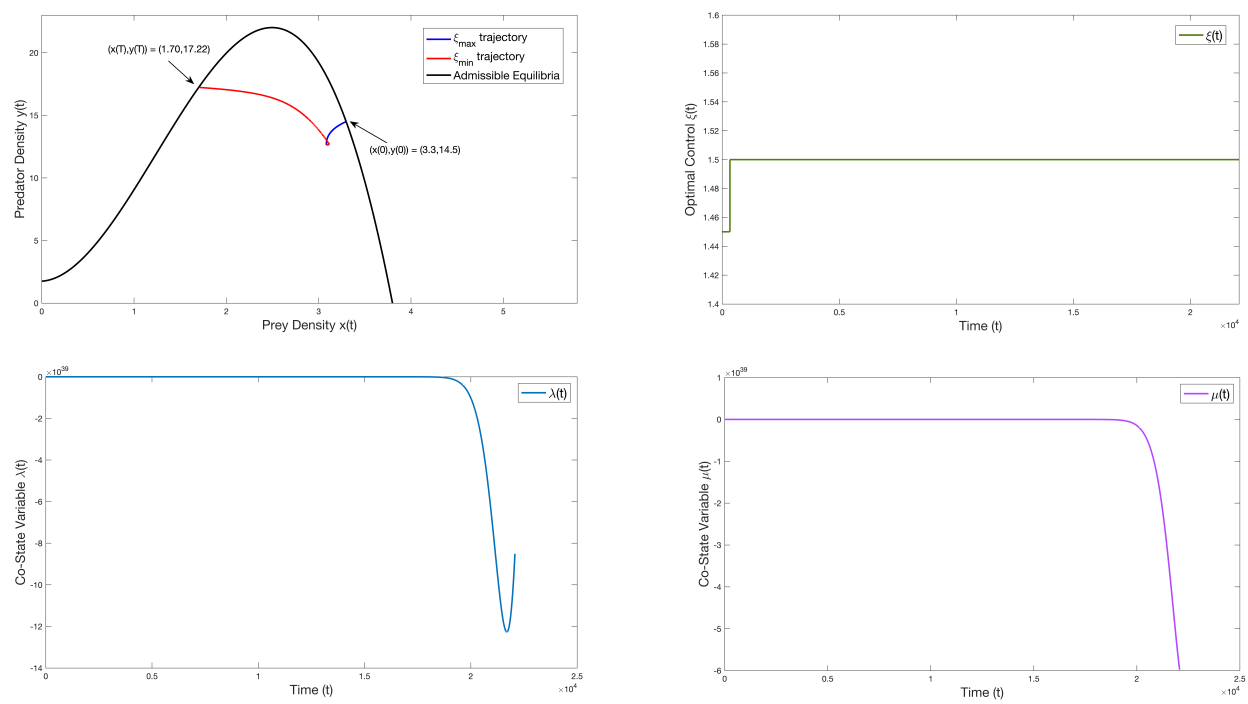

Figure 13: This figure depicts the optimal trajectory of the time optimal control problem (4.1) from the initial state $(3.3,14.5)$ to the terminal state $(1.70,17.22)$ with the parameters values $\gamma=3.8 \beta=2.67, \delta=2.3, \alpha=0.5, \omega=5.5, \xi_{\text {min }}=$ 1.45 , and $\xi_{\max }=1.5$. The initial value chosen for co-state variables is $(\lambda(0), \mu(0))=(, 17)$. This is an example where there is a homoclinic orbit present in the system when $\xi=\xi_{\max }$. However, in the optimal trajectory, we see that the system does not admit a homoclinic orbit for $\xi=\xi_{\min }$. Thus, we can drive the system to its desired terminal state optimally. For $\xi=\xi_{\max }$, we see that both the interior equilibria exist and in fact the initial point chosen for this example is the second equilibrium corresponding to $\xi_{\max }$ which is a saddle leading to a homoclinic orbit that occurs for $\xi<1.5017$. Since $\xi_{\max }=1.5$ in this case and $\xi_{\min }=1.45$ for which the saddle equilibrium and therefore homoclinic orbit do not exist, we can drive the system to the desired terminal state by switching to $\xi_{\min }$. 


\section{Role of Inhibitory Effect}

One of the distinguishing factors that make of the additional food system (1.3) - (1.4) is the type IV functional response. This is due to the inhibitory effect or group defense exhibited by the prey when in large densities. In this section, we will describe some of the unique features and in particular, compare with the type II functional response.

Let $x$ be the dimension-less prey density. Then the non-dimensionalised type IV functional response function is given by

$$
f(x)=\frac{x}{x+(1+\alpha \xi)\left(1+\omega x^{2}\right)}
$$

where the parameters $\alpha$ and $\xi$ represent the quality and quantity of additional food respectively and $\omega$ represents the inhibitory effect displayed by the prey. We see that when $\omega=0$, we get the functional response as

$$
f(x)=\frac{x}{1+\alpha \xi+x}
$$

which is nothing but the type II functional response in the presence of additional food [39]. Also, by com-

\section{Type IV Functional Response}
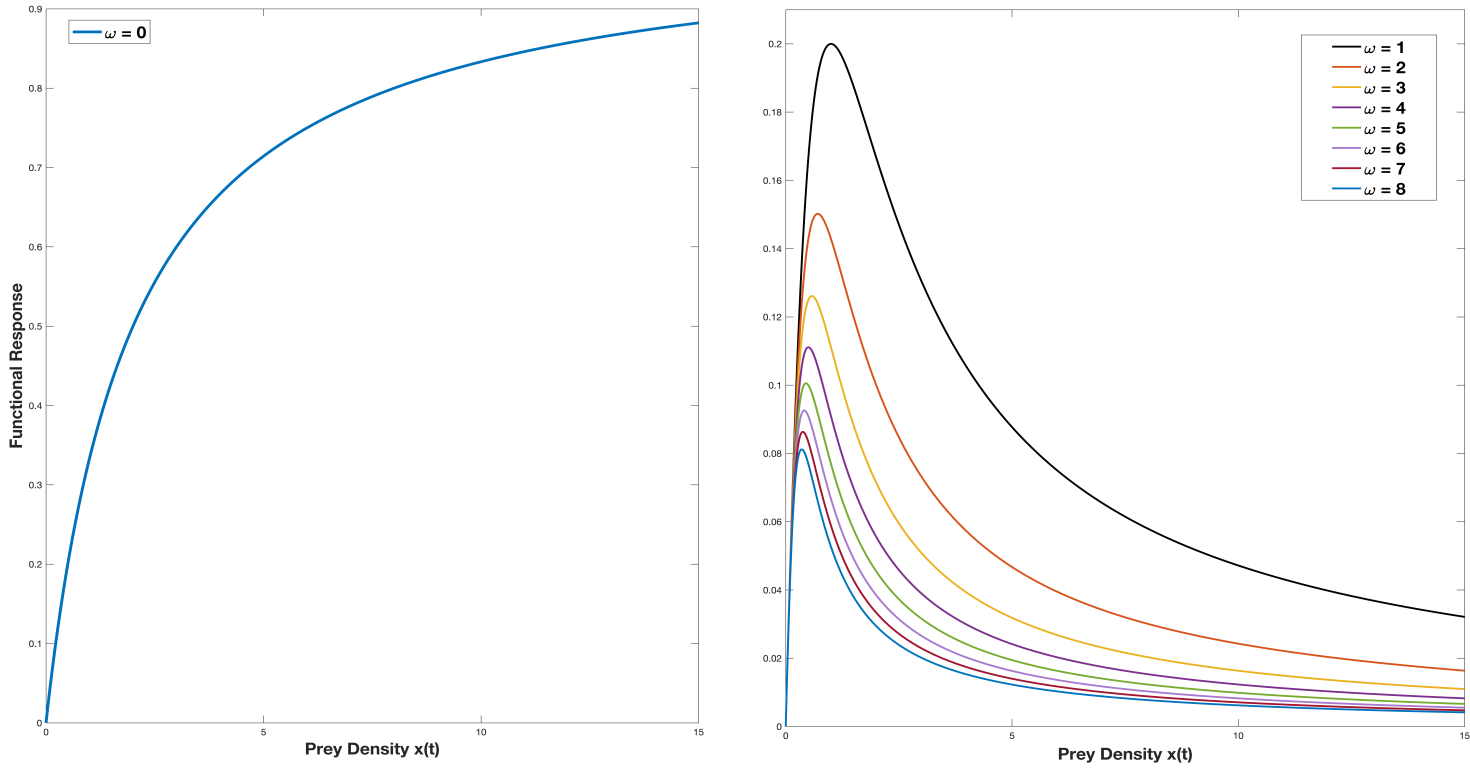

Figure 14: This figure shows the type IV functional response curve with varying inhibitory effect. The first frame on the lest shows the case with $\omega=0$, which is the type II functional response. The second frame shows how the response reduces with increasing prey density as the parameter $\omega$ increases.

paring the expressions (7.1) and (7.2), we see that at low prey densities, both the functional responses behave in a similar manner. We can also observe from the figure 15 below that when $\omega=0$, i.e. when the response is of form type II, the rate of predation increases with increase in prey density and then reaches a saturation. Whereas for type IV response, after a certain prey density, the predation starts to reduce with increase in prey density. To understand this better, we differentiate the functional response with respect to the prey density 
and taking $\frac{d f}{d x}=0$, we get the critical prey density as

$$
x=\frac{1}{\sqrt{\omega}}
$$

From the above expression, we see that the critical prey density reduces with increase in inhibitory effect and as a result, the functional response curve reaches its maximum for lower prey densities and then it starts to decline with increase in prey density. The high predation of predators at low prey densities is similar to the behaviour of the predators that exhibit type II response [39].

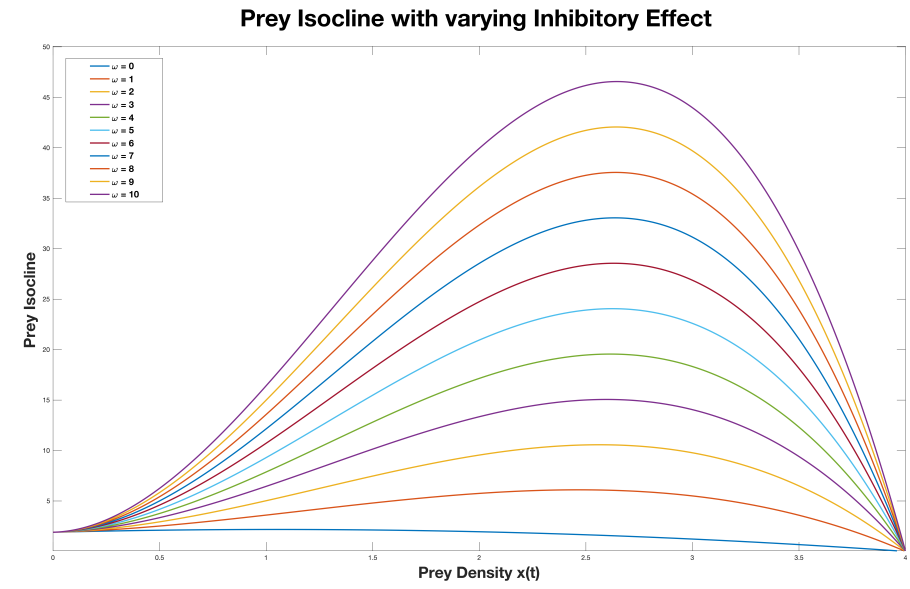

Figure 15: This figure shows the prey isoline curve for the type IV additional food system with varying inhibitory effect.

The parameter $\omega$ plays a crucial role in the nature of prey isocline, on which the dynamics of the system depend on. The prey isocline curve is given by

$$
y=\left(1-\frac{x}{\gamma}\right)\left(x+(1+\alpha \xi)\left(\omega x^{2}+1\right)\right)
$$

Plotting the prey isocline curve (figure - 15) with changing inhibitory effect parameter $\omega$, we see that as $\omega$ increases, the curve moves upwards. This is indicative of the fact that when it intersects the predator isocline, the resultant equilibrium predator population increases with increase in inhibitory effect of the prey. Also, in the absence of inhibitory effect, i.e. for the type II system there is only one interior equilibrium where as for the type IV system, we have two interior equilibria which can exist. Therefore, by incorporating the inhibitory effect of prey into the system with the parameter $\omega$, we see that the system exhibits richer dynamics when compared to the type II system [40, 45] .

Finally, even in the optimal strategy to achieve prey elimination, we see that the inhibitory effect plays a crucial role in achieving the desired terminal state. The figure - 16 depicts the optimal trajectories of the time optimal control problem (4.1) with same boundary conditions and parameters as for figure 9 with increasing inhibitory effect. We have considered 6 different values of inhibitory effect $\omega$. We know that when $\omega=0$, the functional response is nothing but the Type II response. For same parameters with $\omega=0$, results in [38] shows that $y(T)=6.45$ and $T=22.2$. From the various cases presented above, we see that as inhibitory effect increases, the natural enemy density required to eliminate the invasive species increases and the time required to achieve the desired outcome too increases. 




\section{Discussion and Conclusions}

Additional food provided prey-predator systems have been receiving enormous attention over the years by both the theoretical and experimental ecologists as well as mathematicians [3, 15, 31, 36, 46, 45] since the outcomes of these studies are relevant in the biological conservation and bio-control of species in ecosystems. The role of quality and quality of additional food provided to predators and its impact on the ecosystem has also been discussed in various works [2, 30, 31, 33, 48].

Recently, in the works [40] and [45], the authors model and analyze an additional food provided preypredator system that involve type IV functional response. These studies reveal that by providing additional food of suitable quality and quantity to the predators, the system could be driven either to an interior equilibrium ensuring co-existence of species or to an axial equilibrium leading to prey/predator elimination. However, since the terminal states are reached as asymptotes, the practical application of these strategies become difficult. Several ecological studies and experimental observations demonstrate the crucial role played by the quantity of additional food in determining the state and stability of the system [2, 4, 14, 29, 47, 50, 51].

Thus, in this work, to overcome the limitation of asymptotes, we have studied a time optimal control problem to achieve controllability of the system in minimum (finite) time with respect to quantity of additional food. First, we determined the admissible equilibria and the corresponding control necessary to maintain the system at that state. Then, we formulate and study the optimal control problem to drive the system from any initial state to a desired admissible terminal state in minimum time. We have established the existence of optimal solution using the Filippov's Existence Theorem and obtained the characteristics of the optimal control using the Pontryagin's Maximum Principle. Using the Hamiltonian minimization condition, we found the optimal control solution to be of bang-bang type with a possibility of admitting switches in the optimal trajectory in case the objective is to achieve biological conservation. To achieve pest eradication (prey elimination), we found the optimal control the continuous supply of to be of high quantity of additional food.

The findings of this work are in line with some of the experimental observations and are also applicable in the ecological field studies. For example, outcomes of studies in [48] state that increase in volume and concentration of artificial diet sprays results in better pest eradication. This justifies the outcome of achieving prey elimination by providing a constant supply of high quality additional food in large quantity as stated in the results from section 5. Findings from [42] also state that pest elimination could be achieved by optimally providing high quality alternative diet to the generalist predators. However, in order to achieve biological conservation, the strategy of providing high quality of additional food in large quantities is not recommended because it could lead to apparent competition [26] and subsequently prey elimination. Consistent low quality additional food could also lead to predator elimination in some cases [30]. Thus, to achieve co-existence of species, an optimal strategy would be to switch the supply of additional food between the minimum and maximum quantity as stated in the results from section 4.

We have illustrated the theoretical findings numerically by considering various cases. The type IV response is a generalization of the type II response where there is no inhibitory effect in the latter response. One of the important findings of this work is the role of inhibitory effect in achieving bio-control. The numerical illustration (figure 16) in section 7.3 shows that with increasing inhibitory effect, more number of natural enemies are needed to control the pests and also the time taken to achieve the outcome increases. These findings support the experimental observations from [22] which show that increasing inhibitory effect could lead to reduced predation due to large defense mechanism. Thus, the strategy would be to increase natural enemies in order to attack the larger groups of prey species.

The outcomes of this work can be applied to biological conservation and bio-control of species. The illustrations establish the role of inhibitory effect of prey in achieving the desired outcomes. The findings also highlight the importance of quantity of additional food in the global dynamics of the system. These strategies can be implemented by eco-managers in the field studies to achieve desired outcomes in minimum 
time. Proper vigilance and care must be taken while feeding the species because small errors could lead to undesirable outcomes.

Acknowledgements This work is partially funded by the Council of Scientific and Industrial Research - Human Resources Department Group (CSIR-HRDG) under the Direct Senior Research Fellowship (Direct - SRF) scheme with the file number 09/0982(11341)/2021-EMR-I. The authors thank the Journal Editor Shan Zhao and two anonymous reviewers for their valuable comments and suggestions. The first author thanks Prof. B V Rathish Kumar, IIT Kanpur, for giving his time to discuss few topics in Control Theory.

Declaration: The authors declare no conflict of interest.

Dedication: The authors dedicate this paper to the Revered Founder Chancellor of SSSIHL, Bhagawan Sri Sathya Sai Baba. The second author also dedicates this paper to his loving elder brother D. A. C. Prakash who still lives in his heart.

\section{References}

[1] V S Ananth and D. K. K Vamsi. Achieving minimum-time biological conservation and pest eradication for additional food provided predator-prey systems involving inhibitory effect : A qualitative investigation. Acta Biotheoretica (Under Review), 2021.

[2] J Peirce Beach, Livy Williams, Donald L Hendrix, and Leslie D Price. Different food sources affect the gustatory response of anaphes iole, an egg parasitoid of lygus spp. Journal of chemical ecology, 29(5):1203-1222, 2003.

[3] Aleixandre Beltrà, Altea Calabuig, Cristina Navarro-Campos, María José Ramírez-Soria, Antonia Soto, Ferran Garcia-Marí, Felix L Wäckers, and Apostolos Pekas. Provisioning of food supplements enhances the conservation of phytoseiid mites in citrus. Biological Control, 115:18-22, 2017.

[4] Giovanni Benelli, Giulia Giunti, Alejandro Tena, Nicolas Desneux, Alice Caselli, and Angelo Canale. The impact of adult diet on parasitoid reproductive performance. Journal of Pest Science, 90(3):807-823, 2017.

[5] Dana Blackburn, David I Shapiro-Ilan, and Byron J Adams. Biological control and nutrition: Food for thought. Biological Control, 97:131-138, 2016.

[6] Javier Calvo, Karel Bolckmans, Philip A Stansly, and Alberto Urbaneja. Predation by nesidiocoris tenuis on bemisia tabaci and injury to tomato. BioControl, 54(2):237-246, 2009.

[7] Tim Caro. Antipredator defenses in birds and mammals. University of Chicago Press, 2005.

[8] Lamberto Cesari. Optimization theory and applications: problems with ordinary differential equations. Springer Science \& Business Media, 2012.

[9] Colin W Clark. Mathematical bioeconomics- Mathematical Problems in Biology. Springer, 1974.

[10] John B Collings. The effects of the functional response on the bifurcation behavior of a mite predator-prey interaction model. Journal of Mathematical Biology, 36(2):149-168, 1997.

[11] Amartya Das and GP Samanta. Modeling the fear effect on a stochastic prey-predator system with additional food for the predator. Journal of Physics A: Mathematical and Theoretical, 51(46):465601, 2018.

[12] Amartya Das and GP Samanta. Stochastic prey-predator model with additional food for predator. Physica A: Statistical Mechanics and its Applications, 512:121-141, 2018.

[13] Meghadri Das and GP Samanta. A prey-predator fractional order model with fear effect and group defense. International Journal of Dynamics and Control, 9(1):334-349, 2021.

[14] Sarah E Davis, Ruedi G Nager, and Robert W Furness. Food availability affects adult survival as well as breeding success of parasitic jaegers. Ecology, 86(4):1047-1056, 2005.

[15] Joseph S Elkinton, Andrew M Liebhold, and Rose-Marie Muzika. Effects of alternative prey on predation by small mammals on gypsy moth pupae. Population Ecology, 46(2):171-178, 2004.

[16] James D Harwood, Keith D Sunderland, and William OC Symondson. Prey selection by linyphiid spiders: molecular tracking of the effects of alternative prey on rates of aphid consumption in the field. Molecular Ecology, 13(11):3549-3560, 2004.

[17] Mark Kot. Elements of mathematical ecology. Cambridge University Press, 2001.

[18] Henry M Kozak, Robert J Hudson, and Lyle A Renecker. Supplemental winter feeding. Society for Range Management, 1994.

[19] Henry M Kozak, Robert J Hudson, Neil French, and Lyle A Renecker. Winter feeding, lactation, and calf growth in farmed wapiti. Rangelands Archives, 17(14):116-120, 1995. 
[20] Douglas A Landis, Stephen D Wratten, and Geoff M Gurr. Habitat management to conserve natural enemies of arthropod pests in agriculture. Annual review of entomology, 45(1):175-201, 2000.

[21] Daniel Liberzon. Calculus of variations and optimal control theory: a concise introduction. Princeton University Press, 2011.

[22] Melanie McClure and Emma Despland. Defensive responses by a social caterpillar are tailored to different predators and change with larval instar and group size. Naturwissenschaften, 98(5):425-434, 2011.

[23] Gerben J Messelink, Jude Bennison, Oscar Alomar, Barbara L Ingegno, Luciana Tavella, Les Shipp, Eric Palevsky, and Felix L Wäckers. Approaches to conserving natural enemy populations in greenhouse crops: current methods and future prospects. BioControl, 59(4):377-393, 2014.

[24] Sudeshna Mondal and GP Samanta. Dynamics of an additional food provided predator-prey system with prey refuge dependent on both species and constant harvest in predator. Physica A: Statistical Mechanics and its Applications, 534:122301, 2019.

[25] Sudeshna Mondal and GP Samanta. Dynamics of a delayed predator-prey interaction incorporating nonlinear prey refuge under the influence of fear effect and additional food. Journal of Physics A: Mathematical and Theoretical, 53(29):295601, 2020.

[26] CB a Muller and HCJ Godfray. Apparent competition between two aphid species. Journal of Animal Ecology, pages 57-64, 1997.

[27] BSRV Prasad, Malay Banerjee, and PDN Srinivasu. Dynamics of additional food provided predator-prey system with mutually interfering predators. Mathematical biosciences, 246(1):176-190, 2013.

[28] K Durga Prasad and BSRV Prasad. Biological pest control using cannibalistic predators and with provision of additional food: a theoretical study. Theoretical Ecology, 11(2):191-211, 2018.

[29] Kurt Put, Tim Bollens, Felix L Wäckers, and Apostolos Pekas. Type and spatial distribution of food supplements impact population development and dispersal of the omnivore predator macrolophus pygmaeus (rambur)(hemiptera: Miridae). Biological Control, 63(2):172-180, 2012.

[30] RJ Putman and BW Staines. Supplementary winter feeding of wild red deer cervus elaphus in europe and north america: justifications, feeding practice and effectiveness. Mammal Review, 34(4):285-306, 2004.

[31] Stephen M Redpath, Simon J Thirgood, and Fiona M Leckie. Does supplementary feeding reduce predation of red grouse by hen harriers? Journal of Applied Ecology, 38(6):1157-1168, 2001.

[32] Gillian N Robb, Robbie A McDonald, Dan E Chamberlain, and Stuart Bearhop. Food for thought: supplementary feeding as a driver of ecological change in avian populations. Frontiers in Ecology and the Environment, 6(9):479-484, 2008.

[33] Maurice W Sabelis, Paul CJ Van Rijn, et al. When does alternative food promote biological pest control? IOBC WPRS BULLETIN, 29(4):195, 2006.

[34] Banshidhar Sahoo and Swarup Poria. Effects of supplying alternative food in a predator-prey model with harvesting. Applied Mathematics and Computation, 234:150-166, 2014.

[35] Moitri Sen, PDN Srinivasu, and Malay Banerjee. Global dynamics of an additional food provided predator-prey system with constant harvest in predators. Applied Mathematics and Computation, 250:193-211, 2015.

[36] Afsaneh Soltaniyan, Katayoon Kheradmand, Yaghoub Fathipour, and Davoud Shirdel. Supplementation of natural prey with pollen grains exerts an influence on the life table parameters of neoseiulus californicus. Bulletin of Entomological Research, pages 1-7, 2020.

[37] PDN Srinivasu and BSRV Prasad. Time optimal control of an additional food provided predator-prey system with applications to pest management and biological conservation. Journal of mathematical biology, 60(4):591-613, 2010.

[38] PDN Srinivasu and BSRV Prasad. Role of quantity of additional food to predators as a control in predator-prey systems with relevance to pest management and biological conservation. Bulletin of mathematical biology, 73(10):2249-2276, 2011.

[39] PDN Srinivasu, BSRV Prasad, and M Venkatesulu. Biological control through provision of additional food to predators: a theoretical study. Theoretical Population Biology, 72(1):111-120, 2007.

[40] PDN Srinivasu, DKK Vamsi, and I Aditya. Biological conservation of living systems by providing additional food supplements in the presence of inhibitory effect: a theoretical study using predator-prey models. Differential Equations and Dynamical Systems, 26(1):213-246, 2018.

[41] PDN Srinivasu, DKK Vamsi, and VS Ananth. Additional food supplements as a tool for biological conservation of predatorprey systems involving type iii functional response: A qualitative and quantitative investigation. Journal of theoretical biology, 455:303-318, 2018.

[42] Søren Toft. The quality of aphids as food for generalist predators: implications for natural control of aphids. European Journal of Entomology, 68(3):375, 2005.

[43] Pablo Urbaneja-Bernat, Miquel Alonso, Alejandro Tena, Karel Bolckmans, and Alberto Urbaneja. Sugar as nutritional supplement for the zoophytophagous predator nesidiocoris tenuis. BioControl, 58(1):57-64, 2013.

[44] Pablo Urbaneja-Bernat, O Mollá, M Alonso, K Bolkcmans, Alberto Urbaneja, and Alejandro Tena. Sugars as complementary alternative food for the establishment of $\mathrm{n}$ esidiocoris tenuis in greenhouse tomato. Journal of Applied Entomology, 139(3): 161-167, 2015.

[45] DKK Vamsi, Deva Siva Sai Murari Kanumoori, and Bishal Chhetri. Additional food supplements as a tool for biological conservation of biosystems in the presence of inhibitory effect of the prey. Acta biotheoretica, Springer, pages 1-35, 2019. 
[46] Minus Van Baalen, Vlastimil Křivan, Paul CJ van Rijn, and Maurice W Sabelis. Alternative food, switching predators, and the persistence of predator-prey systems. The American Naturalist, 157(5):512-524, 2001.

[47] Bjorn Vandekerkhove and Patrick De Clercq. Pollen as an alternative or supplementary food for the mirid predator macrolophus pygmaeus. Biological Control, 53(2):238-242, 2010.

[48] Mark R Wade, Myron P Zalucki, Steve D Wratten, and Katherine A Robinson. Conservation biological control of arthropods using artificial food sprays: current status and future challenges. Biological control, 45(2):185-199, 2008.

[49] Jeffery T Wilcox and Brendan N Larsen. A group defense incident involving juvenile striped skunks, mephitis mephitis. The Canadian field-naturalist, 122(1):80-82, 2008.

[50] Karin Winkler, Felix L Wäckers, Attila Stingli, and Joop C Van Lenteren. Plutella xylostella (diamondback moth) and its parasitoid diadegma semiclausum show different gustatory and longevity responses to a range of nectar and honeydew sugars. Entomologia Experimentalis et Applicata, 115(1):187-192, 2005.

[51] Bin Zhao, Jian-Wen Qiu, and Pei-Yuan Qian. Effects of food availability on larval development in the slipper limpet crepidula onyx (sowerby). Journal of experimental marine biology and ecology, 294(2):219-233, 2003.

\section{A Appendix - I}

\section{General form of Mayer Problem of Optimal Control}

We will present here the general form of Mayer Problem of Optimal Control as stated in [8]. Let $A$ be a subset of the $t \mathbf{x}$ - space $\mathbb{R}^{1+n}$, let $U$ be a given subset of the $\mathbf{u}$ - space $\mathbb{R}^{m}$. Let $\mathbf{f}(t, \mathbf{x}, \mathbf{u})=\left(f_{1}, f_{2}, \ldots, f_{n}\right)$ be a given function on $A \times U$. For every $(t, \mathbf{x}) \in A$, let $Q(t, \mathbf{x})=\mathbf{f}(t, \mathbf{x}, U) \subset \mathbb{R}^{n}$ be the set of all $z=\left(z_{1}, z_{2}, \ldots, z_{n}\right)$ with $z_{i}=f_{i}(t, \mathbf{x}, \mathbf{u}), i=1,2, \ldots, n$ for some $\mathbf{u} \in U$. Let $B$ be a given subset of $t_{1} \mathbf{x}_{1} t_{2} \mathbf{x}_{2}$ - space $\mathbb{R}^{2 n+2}$. The Mayer problem of optimal control is to find the optimal solution, usually to minimize the functional

$$
I[\mathbf{x}, \mathbf{u}]=g\left(t_{1}, \mathbf{x}\left(t_{1}\right) t_{2} \mathbf{x}\left(t_{2}\right)\right)
$$

for pairs of functions $\mathbf{x}(t)=\left(x_{1}, x_{2}, \ldots, x_{n}\right), \mathbf{u}(t)=\left(u_{1}, u_{2}, \ldots, u_{m}\right), t_{1} \leq t \leq t_{2}, \mathbf{x}$ absolutely continuous, u measurable satisfying

$$
\frac{d \mathbf{x}}{d t}=\mathbf{f}(t, \mathbf{x}(t), \mathbf{u}(t)), t_{1} \leq t \leq t_{2}
$$

boundary conditions

$$
e[\mathbf{x}]=\left(t_{1}, \mathbf{x}\left(t_{1}\right) t_{2} \mathbf{x}\left(t_{2}\right)\right) \in B
$$

and constraints

$$
\begin{aligned}
(t, \mathbf{x}(t)) & \in A, t_{1} \leq t \leq t_{2}, \\
\mathbf{u}(t) & \in U t_{1} \leq t \leq t_{2}
\end{aligned}
$$

in the class $\Omega$ of all admissible pairs $(\mathbf{x}, \mathbf{u})$. By an admissible pair for the problem (A.1) - (A.5) we mean a pair $(\mathbf{x}(t), \mathbf{u}(t)), t_{1} \leq t \leq t_{2}, \mathbf{x}$ absolutely continuous, $\mathbf{u}$ measurable, satisfying all the requirements (A.1) (A.5). Here, $\mathbf{x}$ and $\mathbf{u}$ are also called an admissible trajectory and admissible control respectively.

We will now state the Filippov's Existence Theorem which is used to prove the existence of an optimal solution to the optimal control problem (A.1) - (A.5).

Theorem 4. (The Filippov's Existence Theorem for Mayer Problem of Optimal Control) If A and U are compact, $B$ closed, $\boldsymbol{f}$ is continuous on $A \times U, g$ is continuous on $B, \Omega \neq \Phi$, and for every $(t, \boldsymbol{x}) \in A$ the set $Q(t, \boldsymbol{x})=$ $\boldsymbol{f}(t, \boldsymbol{x}, U) \subset \mathbb{R}^{n}$ is convex, then the objective functional $I[\boldsymbol{x}, \boldsymbol{u}]$ has an absolute minimum in $\Omega$. 